\title{
Model-Order Reduction Using Variational Balanced Truncation with Spectral Shaping
}

\author{
Payam Heydari \\ Department of Electrical Engineering and Computer Science \\ University of California \\ Irvine, CA 92697-2625
}

\author{
Massoud Pedram \\ Department of Electrical Engineering-Systems \\ University of Southern California \\ Los Angeles, CA 90089
}

\begin{abstract}
This paper presents a spectrally-weighted balanced truncation technique for tightly coupled integated circuit interconnects, when the interconnect circuit parameters change as a result of statistical variations in the manufacturing process. The salient features of this algorithm are the inclusion of the parameter variation in the RLC interconnect, the guaranteed passivity of the reduced transfer function, and the availability of provable spectrally-weighted error bounds for the reduced-order system. This paper shows that the variational balanced truncation technique produces reduced systems that accurately follow the time- and frequency-domain responses of the original system when variations in the circuit parameters are taken into consideration. Experimental results show that the new variational spectrally-weighted balanced truncation attains, in average, 30\% more accuracy than the variational Krylov-subspacebased model-order reduction techniques.
\end{abstract}




\section{Introduction}

Model reduction techniques enable circuit designers to capture the interconnect effects with a much shorter computational time than that required for simulation of the full circuit. On the other hand, as the minimum feature sizes shrink to the sub-quarter microns, geometrical variations in the line width, metal height, and dielectric thickness due to process variations have more pronounced effects on the reliability and performance of VLSI circuits [1]. As a consequence, it is crucial to assess the impact of these process variations on model-order reduction techniques.

Among various classes of model reduction techniques, explicit moment-matching algorithms (AWE [2], RICE [3]) and Krylov-subspace-based methods (Pact [4], PVL [5], PRIMA [6]) have been most commonly employed for generating the reduced-order models of the interconnects. The computational complexity of these model-order reduction techniques is primarily due to the matrix-vector products. However, these methods do not provide a provable error bound for the reduced system. Extensions of explicit moment-matching techniques have been proposed recently, to reduce the linear time-varying (LTV) as well as non-linear dynamic systems [7], [8].

An alternative model reduction technique is the balanced realization [9], [10], [11]. While being widely investigated in control system theory, balanced realization techniques have not received the same attention as Krylov-based and Pade-based model reduction techniques in the context of interconnect analysis, partly because these methods involve computation-intensive algorithms [9], [11], [12]. Furthermore, it is well known that the balancing transformation may be poorly conditioned when the system is nearly uncontrollable or unobservable [9]. The most significant drawback of the conventional balanced realization techniques lies in their inability to guarantee a passive reduced-order system. However, the balanced truncation technique can achieve smaller reduced-order models with a better error control than those obtained using Krylov-subspace-based order reduction techniques.

Recently, in [13], the authors developed a guaranteed passive balancing transformation for the model-order reduction of large LTI systems.

The balanced realization-based model reduction methods provide an $L^{\infty}$ error-bound between the transfer function of the original and that of the reduced system. The bottleneck in balanced truncation methods is the computational complexity for solving the Lyapunov equations. Reference [10] uses the truncated balanced realization technique as well as the Schur decomposition to develop an efficient numerical method for the order reduction of a large linear time-invariant (LTI) system. References [11] and [12] propose efficient algorithms to solve the two Lyapunov 
equations in order to obtain the controllability and the observability grammians. The algorithms are based on the Alternated Direction Implicit (ADI) method that was first proposed in [14], [15].

A shortcoming of these model reduction techniques is that they do not reshape the frequency spectrum to emphasize error minimization in some frequency range of interest. Furthermore, they do not address the numerical difficulties when the system is nearly uncontrollable or unobservable. In [16]-[18], a new numerically stable, frequency-weighted balanced truncation technique was presented. The proposed method gives a definite a priori bound on the weighted error, and is guaranteed to be stable even when both input and output weightings are utilized at the same time.

Analyzing the interconnect without taking into account the rather large variations of the interconnect geometries is not useful in practice. These variations are especially large in the inter-layer dielectric (ILD) thickness and the metal line width and height. These process-dependent geometrical variations have a definite impact on the total line and inter-wire coupling parasitics, which in turn results in variations in the signal delay and the coupling noise. In [19], Liu et al. studied the effect of interconnect parameter variations on the Krylov-subspace model-order reduction techniques. The paper adds the variability to the Krylov-subspace-based model reduction method [4], [6] by borrowing some ideas from the matrix perturbation theory [20]. The authors allow twodimensional variations on the projection matrices. To compute the corresponding sensitivities of the susceptance and conductance matrices to each dimensional variation, some sample points were picked up and the dominant eigenvalues/eigenvectors were calculated.

The goal of the present paper is to study the effects of process variations and spectral shaping on model-order reduction using an extended balanced truncation technique and to propose an efficient order reduction technique that includes these effects. The main contribution of this paper is the use of the spectrally-weighted balanced truncation method proposed in [16]-[18] combined with a new variational balanced truncation approach proposed in that accounts for process variations resulting in a new variational spectrally-weighted balanced truncation method [21]. The works of [18], [19], and [21] have laid the groundwork for interesting research on various extensions of balanced truncation method (e.g., [13]) and model-order reduction of parameterized interconnects (e.g., [22]). To preserve the passivity, the proposed algorithm employs results of [13]. As the future work, we will investigate the systematic way of determining the input/output weighting functions for the spectrally-weighted balanced truncation method.

This paper is organized as follows: Section 2 gives a brief overview of the balanced realization technique. Section 3 describes the frequency-weighted model reduction proposed in [16]-[18]. A discussion about the effect of process variations on interconnect modeling is provided in Section 
4. Also, in Section 4, the variational balanced truncation with spectral shaping is illustrated, and a theoretical comparison between the proposed algorithm and the work presented in [19] is provided. Section 5 discusses the experimental results including the comparison between the proposed model reduction technique and different model-order reduction techniques in [6] and [13]. The accuracy of the proposed order reduction technique in generating low-order reduced systems for electromagnetically coupled global interconnect and H-tree clock distribution network is examined. Finally, Section 6 presents the concluding remarks for this paper.

\section{An overview of the balanced realization technique}

Consider the state-space representation of a linear time-invariant (LTI) system:

$$
\begin{gathered}
\dot{\mathbf{x}}=\mathbf{A} \mathbf{x}+\mathbf{B u} \\
\mathbf{y}=\mathbf{C x}
\end{gathered}
$$

where $\mathbf{A} \in \mathfrak{R}^{n \times n}, \mathbf{B} \in \mathfrak{R}^{n \times p}, \mathbf{C} \in \mathfrak{R}^{p \times n}$, and $\mathbf{u} \in \mathfrak{R}^{p \times 1} . \mathbf{x} \in \mathfrak{R}^{n \times 1}$ represents the state vector of the system. $n$ denotes the order of the system, and $p$ represents the size of the input vector. In a general nth-order RLC circuit, for example, the state vector constitutes node voltages across circuit capacitances, voltage sources, currents flowing through inductors, and current sources. The system matrix $\mathbf{A}$ is readily obtained from the conductance and susceptance matrices of the RLC circuit. Similar to [5], the representation of $\mathbf{A}$ with respect to the susceptance, $L \in \mathfrak{R}^{n \times n}$, and conductance, $G \in \mathfrak{R}^{n \times n}$, matrices is carried out around an expansion point $\left(s_{0} \neq 0\right) \in \mathrm{C}$, such that $\mathbf{A}=-\left[\left(L+G / s_{0}\right)^{-1} G\right]$ becomes non-singular. The goal of the model-order reduction is to obtain a similar lower order system

$$
\begin{gathered}
\mathbf{x}_{r}=\mathbf{A}_{r} \mathbf{x}_{r}+\mathbf{B}_{r} \mathbf{u}_{r} \\
\mathbf{y}_{r}=\mathbf{C}_{r} \mathbf{x}_{r}
\end{gathered}
$$

where $\mathbf{A}_{r} \in \mathfrak{R}^{k \times k}, \mathbf{B}_{r} \in \mathfrak{R}^{k \times p}, \mathbf{C}_{r} \in \mathfrak{R}^{p \times k}, \mathbf{u}_{r} \in \mathfrak{R}^{p \times 1}$, and $\mathbf{x}_{r} \in \mathfrak{R}^{k \times 1} . r$, the order of the reduced system, is much smaller than that of the original system. The balanced truncation technique carries out the order reduction by providing an $L^{\infty}$ error-bound between the transfer functions of the original and the reduced systems.

Central to the state-space description and balanced truncation of dynamic systems are the controllability and observability grammians. The key functions in formulating the passivity-preserving balanced truncation of linear systems are the positive-definite controllability and observability grammians $\mathbf{P}_{L}$ and $\mathbf{Q}_{L}$ of the system, which are obtained by solving Lur'e equations [13], i.e., 


$$
\begin{gathered}
\mathbf{A} \mathbf{P}_{L}+\mathbf{P}_{L} \mathbf{A}^{T}=-\mathbf{K}_{c} \mathbf{K}_{c}^{T} \\
\mathbf{P}_{L} \mathbf{C}^{T}-\mathbf{B}=-\mathbf{K}_{c} \mathbf{J}_{c}^{T} \\
\mathbf{J}_{c} \mathbf{J}_{c}^{T}=\mathbf{D}+\mathbf{D}^{T} \\
\mathbf{A}^{T} \mathbf{Q}_{L}+\mathbf{Q}_{L} \mathbf{A}=-\mathbf{K}_{o}^{T} \mathbf{K}_{o} \\
\mathbf{Q}_{L} \mathbf{B}-\mathbf{C}^{T}=-\mathbf{K}_{o}^{T} \mathbf{J}_{o} \\
\mathbf{J}_{o}^{T} \mathbf{J}_{o}=\mathbf{D}+\mathbf{D}^{T}
\end{gathered}
$$

The controllability grammian is a measure of how much the input energy is coupled to the states of the system. The observability grammian is a measure of how the states and the outputs are coupled to each other. The controllability and observability grammians give some interesting insights about the system characteristics. It can be proved ( $c f$. reference [9]) that there exists a similarity transformation matrix $\mathbf{T}$ that maps any given representation of a system to a balanced realization such that the controllability and observability grammians of the new system $\left(\mathbf{A}_{r}, \mathbf{B}_{r}, \mathbf{C}_{r}\right)$ are equal and diagonal, i.e.,

$$
\mathbf{P}_{L, r}=\mathbf{Q}_{L, r}=\Sigma=\operatorname{diag}\left(\xi_{1}, \xi_{2}, \ldots, \xi_{n}\right)
$$

where $\xi_{1} \geq \xi_{2} \geq \ldots \geq \xi_{n}>0$. Calculating the diagonal matrices $\mathbf{P}_{L, r}$ and $\mathbf{Q}_{L, r}$ whose diagonal elements represent the singular values associated with each state variable $x_{k}, 1 \leq k \leq n$; makes it possible to find a criterion for evaluating the possibility of eliminating $x_{k}$ in the reduced model scheme while preserving the time and frequency domain characteristics of the original system. The matrix $\Sigma$ is partitioned into to two submatrices:

$$
\Sigma=\left[\begin{array}{cc}
\Sigma_{1} & 0 \\
0 & \Sigma_{2}
\end{array}\right]
$$

where $\Sigma_{1} \in \mathfrak{R}^{k \times k}, \Sigma_{2} \in \mathfrak{R}^{(n-k) \times(n-k)}$, and the new coordinate transformed system $\left(\mathbf{A}_{r}, \mathbf{B}_{r}, \mathbf{C}_{r}\right)$ is also partitioned in conformity with $\Sigma$ as:

$$
\mathbf{A}_{r}=\mathbf{T A T}^{-1}=\left[\begin{array}{ll}
\mathbf{A}_{11} & \mathbf{A}_{12} \\
\mathbf{A}_{21} & \mathbf{A}_{22}
\end{array}\right], \quad \mathbf{B}_{r}=\mathbf{T B}=\left[\begin{array}{l}
\mathbf{B}_{1} \\
\mathbf{B}_{2}
\end{array}\right], \quad \mathbf{C}_{r}=\mathbf{C} \mathbf{T}^{-1}=\left[\begin{array}{ll}
\mathbf{C}_{1} & \mathbf{C}_{2}
\end{array}\right]
$$

The reduced-order model based on $\left(\mathbf{A}_{11}, \mathbf{B}_{1}, \mathbf{C}_{1}\right)$ is stable and the $L^{\infty}$-error is bounded by:

$$
\left\|\mathbf{H}(s)-\mathbf{H}_{r}^{k}(s)\right\|_{\infty} \leq 2\left(\sum_{i=k+1}^{n} \xi_{i}\right)
$$


[13] has proved that $\mathbf{H}_{r}^{k}(s)$ obtained using this method is actually positive-real, and therefore, the reduced-order model is passive. According to Eq. (10), the error bound is similar to the twicethe-sum-of-the-tail formula obtained for conventional balanced truncation method presented in [9]-[12]. The balanced realization relies on the calculation of controllability and observability grammians of the original system. The Lur'e equations are solved in order to obtain the system grammians. The procedure to calculate the grammians effectively involves applying the Cholesky factorization to matrix $\mathbf{Q}[10]$,

$$
\mathbf{Q}=\mathbf{R}^{T} \mathbf{R}
$$

and diagonalizing the matrix $\mathbf{R P R}^{T}$, i.e.,

$$
\mathbf{R} \mathbf{P} \mathbf{R}^{T}=\mathbf{U} \Sigma^{2} \mathbf{U}^{T} \quad \text { with } \mathbf{U}^{T} \mathbf{U}=\mathbf{I}
$$

A balancing transformation, $\mathbf{T}$, which maps the original system to the balanced realization form is obtained as follows:

$$
\mathbf{T}=\Sigma^{-1 / 2} \mathbf{U}^{T} \mathbf{R}
$$

This algorithm, however, involves solving a generalized eigenvalue problem whose dimension is as large as the order of the original transfer function [9]. More precisely, the Lur'e equations (which is similar in form to Lyapunov equations) still need to be solved for the mapped system in order to obtain the coordinate-mapped controllability and observability grammians, $\mathbf{P}_{L}$ and $\mathbf{Q}_{L}$, a task that is computationally quite expensive.

The problem of solving a high-order set of matrix equations for the eigenvalues and eigenvectors of the system can be avoided by using the Krylov subspace-based methods. In fact, studying the algorithm proposed by [23] shows that it is only necessary to find the first $k$ largest eigenvalues of the product and their corresponding left and right eigenvectors. Based on this observation, a modified version of the Safanov's algorithm is used in [10]. More precisely, in [10], the Arnoldi algorithm is utilized to compute the largest eigenvalues and the corresponding left and right eigenvectors. $\mathbf{P}_{L} \mathbf{Q}_{L}$ is a large symmetric matrix, which is a positive definite matrix.

The underlying problem is to efficiently obtain the first $k$ largest eigenvalues of a symmetric matrix. The Lanczos method is utilized to efficiently calculate this subset of the system eigenvalues. The Lanczos algorithm reduces the original large symmetric matrix, $\mathbf{M}=\mathbf{P}_{L} \mathbf{Q}_{L}$, to a smaller tridiagonal matrix $\mathbf{T}_{q}$ where $\mathbf{T}_{q} \in \mathfrak{R}^{q \times q}$. The algorithm involves successfully filling in the columns $\mathbf{V}_{L}$ and $\mathbf{V}_{R}$ such that $\mathbf{V}_{L}^{\mathrm{T}} \mathbf{V}_{R}=\mathrm{D}_{q}=\operatorname{diag}\left(\delta_{1}, \delta_{2}, \ldots, \delta_{q}\right)$, where $\mathbf{V}_{L}=\left[\mathbf{v}_{L 1} \mathbf{v}_{L 2} \ldots \mathbf{v}_{L q}\right]$ and $\mathbf{V}_{R}=$ 
$\left[\mathbf{v}_{R 1} \mathbf{v}_{R 2} \ldots \mathbf{v}_{R q}\right]$ and the vectors $\left\{\boldsymbol{v}_{R i}\right\}_{i=1}^{q}$ and $\left\{\boldsymbol{v}_{L i}\right\}_{i=1}^{q}$ span the Krylov subspaces $K_{q}\left(\mathbf{v}_{1}\right.$, $\left.\mathbf{P}^{(\mathrm{L})} \mathbf{Q}^{(\mathrm{L})}\right)$ and $K_{q}\left(\mathbf{w}_{1}, \mathbf{P}^{(\mathrm{L})} \mathbf{Q}^{(\mathrm{L})}\right)$, respectively:

$$
\begin{gathered}
\operatorname{colsp}\left(\mathbf{V}_{L}\right)=K_{q}\left(\boldsymbol{v}_{L 1}, \mathbf{M}\right)=\operatorname{span}\left\{\boldsymbol{v}_{L 1}, \mathbf{M} \boldsymbol{v}_{L 1}, \ldots, \mathbf{M}^{q-1} \boldsymbol{v}_{L 1}\right\} \\
\operatorname{colsp}\left(\mathbf{V}_{R}\right)=K_{q}\left(\boldsymbol{v}_{R 1}, \mathbf{M}\right)=\operatorname{span}\left\{v_{R 1}, \mathbf{M} v_{R 1}, \ldots, \mathbf{M}^{q-1} \boldsymbol{v}_{R 1}\right\} \\
\mathbf{V}_{L}^{T}\left(\mathbf{P}^{(L)} \mathbf{Q}^{(L)}\right) \mathbf{V}_{R}=\mathbf{T}_{q}=\left[\begin{array}{ccccc}
\alpha_{1} & \beta_{2} & 0 & \ldots & 0 \\
\gamma_{2} & \alpha_{2} & \beta_{3} & \ddots & \vdots \\
0 & \gamma_{3} & \ddots & \ddots & 0 \\
\vdots & \ddots & \ddots & \ddots & \beta_{q} \\
0 & \ldots & 0 & \gamma_{q} & \alpha_{q}
\end{array}\right] \\
\mathbf{V}_{L}^{T} \mathbf{V}_{R}=\mathbf{D}_{q}=\operatorname{diag}\left(\delta_{1}, \ldots, \delta_{q}\right)
\end{gathered}
$$

The columns of the two projection matrices $\mathbf{V}_{L}, \mathbf{V}_{R} \in \mathfrak{R}^{n \times q}$, form bases for the respective right and left eigenspaces of the Krylov subspace in the Lancsoz method, associated with their "big" eigenvalues $\xi_{1}^{2}, \ldots, \xi_{q}^{2}$. The matrices $\mathbf{V}_{L}$ and $\mathbf{V}_{R}$ are used as bases for the relevant eigenspaces of the matrix $\mathbf{P}_{L} \mathbf{Q}_{L}$ in the derivation of the reduced-order model.

A shortcoming of the conventional balanced truncation techniques proposed in [9], [11]-[12], and [13] is that they do not reshape the frequency spectrum to emphasize error minimization in some frequency range of interest. Our preliminary experiments show that the error between the transfer function of the actual interconnect system and the transfer function of the reduced order system obtained using any conventional order reduction technique is frequency-dependent, and increases with frequency, which is undesirable. Fig. 1 visualizes this observation, where the energy of the error arising from order reduction of the original system is frequency dependent.

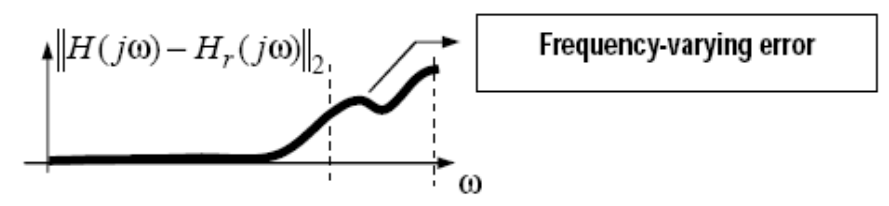

Fig. 1. The $\mathrm{L}_{2}$-norm of the error vs. frequency

Section 3 describes a numerically stable, frequency-weighted balanced truncation technique proposed by [16]-[18]. 


\section{Balanced truncation with spectral shaping}

So far, it has been observed that balanced realization is an attractive model reduction technique due to the fact that it provides a priori $L^{\infty}$-error bound for the reduced-order system. As mentioned above, the frequency dependence of the error between the reduced-order and the original system transfer functions is important in many applications. In other words, error should be small in one or more frequency ranges of interests, while it can be larger in other ranges, depending on the application. The balanced truncation technique extended to include weighting on the input and/or output as shown in Fig. 2 [16]-[18].

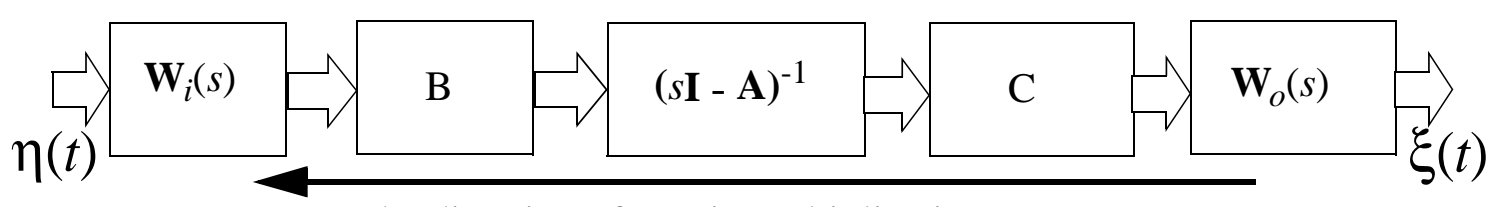

The direction of matrix multiplication

Fig. 2. Block diagram of the original LTI system along with input and output weightings

To determine the state-space characteristics of the new augmented system, two basic questions must be addressed ( $c f$. Fig. 2):

1. What set of points in the $x$-state space could be a part of the zero initial condition response for the weighted input denoted by $\eta(t)$ ?

2. What set of points in the $x$-state space as initial conditions could produce a weighted output denoted by $\xi(t)$ ?

Consider the state-space representation of a set of tightly coupled RLC interconnects given by equations (1) and (2). The goal of the frequency-weighted balanced realization technique is to calculate $\mathbf{H}_{r}^{k}(s)$ of degree $k(k<n)$, while minimizing

$$
\left\|\mathbf{W}_{o}(s)\left(\mathbf{H}(s)-\mathbf{H}_{r}^{k}(s)\right) \mathbf{W}_{i}(s)\right\|_{\infty}
$$

To obtain such a reduced system, the grammians of the augmented system must first be calculated and the same steps that are used for a unity-weighted system should then be employed. Most importantly, the weighting functions are chosen to be positive real functions. Note that the purpose of reshaping the frequency response is to minimize the error of the reduced order system. The weighting functions should thus: (1) have a simple rational function in the s-domain, so that both the frequency- and time-domain behavior of the augmented system can be easily studied, and (2) be synthesized using simple low-order passive RLCK, in case the actual realization is of interest. 
First, we write the Laplace transformation of the input and output weighting functions that are chosen to be stable functions [16]:

$$
\begin{aligned}
& \mathbf{W}_{i}(s)=\mathbf{C}_{i}\left(s \mathbf{I}-\mathbf{A}_{i}\right)^{-1} \mathbf{B}_{i}+\mathbf{D}_{i} \\
& \mathbf{W}_{o}(s)=\mathbf{C}_{o}\left(s \mathbf{I}-\mathbf{A}_{o}\right)^{-1} \mathbf{B}_{o}+\mathbf{D}_{o}
\end{aligned}
$$

According to the definition in [24], the controllable subspace of the augmented system, $\mathbf{H}(s) \mathbf{W}_{i}(s)$, is the solution set to the first question. A controller-form realization of the augmented system $\mathbf{H}(s) \mathbf{W}_{i}(s)$ is as follows:

$$
\overline{\mathbf{A}_{i}}=\left[\begin{array}{cc}
\mathbf{A} & \mathbf{B C}_{i} \\
\mathbf{0} & \mathbf{A}_{i}
\end{array}\right], \overline{\mathbf{B}}_{i}=\left[\begin{array}{c}
\mathbf{B D}_{i} \\
\mathbf{B}_{i}
\end{array}\right], \overline{\mathbf{C}_{i}}=\left[\begin{array}{ll}
\mathbf{C} & \mathbf{0}
\end{array}\right]
$$

Similarly, according to the definition in [24], the observable subspace of the augmented system, $\mathbf{W}_{o}(s) \mathbf{H}(s)$, is the solution set to the second question. An observer-form realization of the augmented system $\mathbf{W}_{o}(s) \mathbf{H}(s)$ is as follows:

$$
\overline{\mathbf{A}}_{o}=\left[\begin{array}{cc}
\mathbf{A} & \mathbf{0} \\
\mathbf{B}_{o} \mathbf{C} & \mathbf{A}_{o}
\end{array}\right], \overline{\mathbf{B}}_{o}=\left[\begin{array}{l}
\mathbf{C} \\
\mathbf{0}
\end{array}\right] \quad, \overline{\mathbf{C}}_{o}=\left[\begin{array}{ll}
\mathbf{D}_{o} \mathbf{C} & \mathbf{C}_{o}
\end{array}\right]
$$

For a complete explanation of controller and observer form realizations, see chapter 3 of reference [24]. Since all controllable and observable modes of these two augmented systems are determined by $n \times n$ upper left corner submatrices of $\overline{\mathbf{A}_{i}}$ and $\overline{\mathbf{A}_{o}}$, the desired controllability and observability grammians are thus given by the corresponding $n \times n$ upper left corner submatrices of $\overline{\mathbf{P}}$ and $\overline{\mathbf{Q}}$ :

$$
\overline{\mathbf{P}}_{L}=\left[\begin{array}{cc}
\mathbf{P} & \mathbf{P}_{12} \\
\mathbf{P}_{12}^{T} & \mathbf{P}_{22}
\end{array}\right], \quad \overline{\mathbf{Q}}_{L}=\left[\begin{array}{ll}
\mathbf{Q} & \mathbf{Q}_{12} \\
\mathbf{Q}_{12}^{T} & \mathbf{Q}_{22}
\end{array}\right]
$$

where $\overline{\mathbf{P}}$ and $\overline{\mathbf{Q}}$ must satisfy the following Lur'e equations:

$$
\begin{aligned}
\overline{\mathbf{A}}_{i} \overline{\mathbf{P}}_{L}+\overline{\mathbf{P}}_{L} \overline{\mathbf{A}}_{i}^{T}+\overline{\mathbf{K}}_{c, i} \overline{\mathbf{K}}_{c, i}^{T}=\mathbf{0} \\
\overline{\mathbf{P}}_{L} \overline{\mathbf{C}}_{i}^{T}-\overline{\mathbf{B}}_{i}=-\overline{\mathbf{K}}_{c, i} \mathbf{J}_{c, i}^{T} \\
\mathbf{J}_{c, i} \mathbf{J}_{c, i}^{T}=\mathbf{D}+\mathbf{D}^{T} \\
\overline{\mathbf{A}}_{o}^{T} \overline{\mathbf{Q}}_{L}+\overline{\mathbf{Q}}_{L} \overline{\mathbf{A}}_{o}+\overline{\mathbf{K}}_{o, o}^{T} \overline{\mathbf{K}}_{o, o}=\mathbf{0} \\
\overline{\mathbf{Q}}_{L} \overline{\mathbf{B}}_{o}-\overline{\mathbf{C}}_{o}^{T}=-\overline{\mathbf{K}}_{o, o}^{T} \mathbf{J}_{o, o} \\
\mathbf{J}_{o, o}^{T} \mathbf{J}_{o, o}=\mathbf{D}_{o}+\mathbf{D}_{o}^{T}
\end{aligned}
$$


The passivity of the original system and positive-realness of weighting function guarantee the existence of matrices $\overline{\mathbf{P}}_{L}, \overline{\mathbf{Q}}_{L} \geq 0, \mathbf{J}_{c, i}$, and $\mathbf{J}_{o, o}$, which satisfy equations (19) and (20) [13]. Expanding the $n \times n$ upper left corner block of the Lur'e equations yields:

$$
\begin{gathered}
\mathbf{A} \mathbf{P}+\mathbf{P} \mathbf{A}^{T}+\overline{\mathbf{K}}_{c 1} \overline{\mathbf{K}}_{c 1}^{T}=\mathbf{0} \\
\mathbf{P} \mathbf{C}^{T}-\mathbf{B} \mathbf{D}_{i}=-\overline{\mathbf{K}}_{c 1} \mathbf{J}_{c 1}^{T} \\
\mathbf{J}_{c 1} \mathbf{J}_{c 1}^{T}=\mathbf{D}+\mathbf{D}^{T} \\
\mathbf{A}^{T} \mathbf{Q}+\mathbf{Q} \mathbf{A}+\overline{\mathbf{K}}_{o 1}^{T} \overline{\mathbf{K}}_{o 1}=\mathbf{0} \\
\mathbf{Q B}-\mathbf{C}^{T} \mathbf{D}_{o}^{T}=-\overline{\mathbf{K}}_{o 1}^{T} \mathbf{J}_{o 1} \\
\mathbf{J}_{o 1}^{T} \mathbf{J}_{o 1}=\mathbf{D}_{o}+\mathbf{D}_{o}^{T}
\end{gathered}
$$

We define the new variables:

$$
\begin{gathered}
\mathbf{X}=\overline{\mathbf{K}}_{c 1} \overline{\mathbf{K}}_{c 1}^{T}=\mathbf{B C}_{i} \mathbf{P}_{12}+\mathbf{P}_{12}^{T} \mathbf{C}_{i}^{T} \mathbf{B}^{T}+\overline{\mathbf{K}}_{c, i} \overline{\mathbf{K}}_{c, i}^{T} \\
\mathbf{Y}=\overline{\mathbf{K}}_{o 1}^{T} \overline{\mathbf{K}}_{o 1}=\mathbf{Q}_{12} \mathbf{B}_{o} \mathbf{C}+\mathbf{C}^{T} \mathbf{B}_{o}^{T} \mathbf{Q}_{12}^{T}+\overline{\mathbf{K}}_{o, o}^{T} \overline{\mathbf{K}}_{o, o}
\end{gathered}
$$

It is readily seen that $\mathbf{X}$ and $\mathbf{Y}$ are symmetric matrices. As a consequence, there exist orthogonal matrices $\mathbf{U}$ and $\mathbf{V}$ and diagonal matrices $\mathbf{S}$ and $\mathbf{Z}$ such that:

$$
\begin{aligned}
& \mathbf{X}=\mathbf{U S U}^{T} \\
& \mathbf{Y}=\mathbf{V} \mathbf{Z} \mathbf{V}^{T}
\end{aligned}
$$

where $\mathbf{S}=\operatorname{diag}\left(s_{1}, s_{2}, \ldots, s_{n}\right)$, and $\mathbf{Z}=\operatorname{diag}\left(z_{1}, z_{2}, \ldots, z_{n}\right)$, and $\left|s_{1}\right| \geq\left|s_{2}\right| \geq \ldots \geq\left|s_{n}\right| \geq 0$, $\left|z_{1}\right| \geq\left|z_{2}\right| \geq \ldots \geq\left|z_{n}\right| \geq 0$. Suppose that $\operatorname{rank}(\mathbf{X})=i$ and $\operatorname{rank}(\mathbf{Y})=j$, where $1 \leq i, j \leq n$. We can write:

$$
\begin{aligned}
& \overline{\mathbf{B}}=\mathbf{U} \operatorname{diag}\left(\left|s_{1}\right|^{1 / 2}, \ldots,\left|s_{i}\right|^{1 / 2}, 0, \ldots, 0\right) \\
& \overline{\mathbf{C}}=\operatorname{diag}\left(\left|z_{1}\right|^{1 / 2}, \ldots,\left|z_{j}\right|^{1 / 2}, 0, \ldots, 0\right) \mathbf{V}^{T}
\end{aligned}
$$

Now, suppose that $\overline{\mathbf{K}}_{c 1}$ and $\overline{\mathbf{K}}_{o 1}$ is uniquely specified as $\overline{\mathbf{K}}_{c 1}=\overline{\mathbf{B}}, \overline{\mathbf{K}}_{o 1}=\overline{\mathbf{C}}$, and moreover, let $\hat{\mathbf{P}}$ and $\hat{\mathbf{Q}}$ denote the solutions of the Lur'e equations (21) and (22), which are both positive semi-definite [13]. The similarity transformation $\hat{\mathbf{T}}$ that simultaneously diagonalizes $\hat{\mathbf{P}}$ and $\hat{\mathbf{Q}}$ is thus as follows:

$$
\hat{\mathbf{T}} \hat{\mathbf{P}} \hat{\mathbf{T}}^{T}=\left(\hat{\mathbf{T}}^{-1}\right)^{T} \hat{\mathbf{Q}} \hat{\mathbf{T}}^{-1}=\operatorname{diag}\left(\xi_{1}, \ldots, \xi_{r}, \xi_{r+1}, \ldots, \xi_{n}\right)
$$


Similar to the unit-weighted balanced truncation method, this transformation matrix, $\hat{\mathbf{T}}$, is used to map the original system to a new coordinate transformed system for which the controllability and observability grammians are diagonal and identical. The reduced-order system is then obtained from the transformed system. Most importantly, the reduced-order system is passive, because $\hat{\mathbf{T}}$ has been constructed using the positive semi-definite grammians $\hat{\mathbf{P}}$ and $\hat{\mathbf{Q}}$. The balanced truncation algorithm based on the coordinate transformed system using $\hat{\mathbf{T}}$ preserves the positive-realness of the transfer function, and hence preserves passivity. Note that $\hat{\mathbf{T}}$ contains the characteristics of the weighting functions. The input and output weightings are determined based on the range of frequencies where the maximum accuracy is desired. The weighting functions should emphasize the frequency ranges where more accuracy is required. Similarly, they must deemphasize the range of frequencies where the noise resulting from the order reduction has very minimal energy or is out of the desired frequency bound.

\section{Variational spectrally-weighted balanced truncation}

Due to process variations, interconnect technology parameters are varying substantially. These parameters can have as much as a $30 \%$ variation off their nominal values [1]. Therefore, the effect of the process variations on the interconnect delay and crosstalk should be taken into consideration. A common approach to anticipate these variations in the design is the conventional skew-corner, worst-case modeling. This method, however, is too conservative because the probability of all 3- $\sigma$ process corner values occurring simultaneously is very small. As a consequence, statistically-based worst-case interconnect modelings using Monte-carlo simulation have been proposed [25], [26]. These approaches, however, fail to handle large circuits that exist in reality. To alleviate the problem of having large computational complexity (as also mentioned in [19]) the effect of process variations must be taken into account in model-order reduction algorithms. Furthermore, the resulting variational reduced-order model needs to converge to the reduced-order model of the nominal network when all the parameter variations are zero.

Characterization of the interconnect geometry variation is an important issue in deep-submicron VLSI technology. In order to accurately assess the performance of a complex interconnect structure, it is essential to characterize the interconnect geometry, which in turn specifies the interconnect parasitics [19].

From a designer's point of view, one important source of the IC performance variability is the physical source of variability [27]. The manufacturing process variations can be overlooked at 
two levels of IC fabrication. The variation at the first level of IC fabrication includes the case where the interconnect (or device) parameters are constant within a die but vary within a wafer or a lot. The process variation at the second level results in the variations of the device and interconnect parameters within the die. Details about the physical imperfections, which lead to each of these variations, are beyond the scope of this paper (see [25]-[27]). These two variations are uncorrelated, thereby simplifying the mathematical formulations. Moreover, The physical variations induced by non-idealities of the manufacturing process manifest themselves to electrical variations. For instance, for a given on-chip metal wire, a within-the-die width variation of $w_{1, d}$, a within-the-wafer variation of $w_{1, w}$, a within-the-die height variation of $w_{2, d}$, and within-thewafer height variation of $w_{1, w}$ will result in the non-zero offsets for the parasitic elements associated with that metal wire, i.e.,

$$
\begin{gathered}
r\left(w_{1}, w_{2}\right)=r_{0}+\Delta r_{1, d} w_{1, d}+\Delta r_{1, w} w_{1, w}+\Delta r_{2, d} w_{2, d}+\Delta r_{2, w} w_{2, w}=r_{0}+\Delta r \\
c\left(w_{1}, w_{2}\right)=c_{0}+\Delta c_{1, d} w_{1, d}+\Delta c_{1, w} w_{1, w}+\Delta c_{2, d} w_{2, d}+\Delta c_{2, w} w_{2, w}=c_{0}+\Delta c \\
l\left(w_{1}, w_{2}\right)=l_{0}+\Delta l_{1, d} w_{1, d}+\Delta l_{1, w} w_{1, w}+\Delta l_{2, d} w_{2, d}+\Delta l_{2, w} w_{2, w}=l_{0}+\Delta l
\end{gathered}
$$

where $r_{0}, c_{0}, l_{0}$ are the zero-offset parasitic resistance, capacitance, and inductance per-unit length. In general, for the susceptance and conductance matrices of the interconnect system that are exposed to the process variations, we have:

$$
\begin{gathered}
G_{\text {var }}\left(w_{1}, w_{2}\right)=G_{0}+\Delta G_{1} w_{1}+\Delta G_{2} w_{2}=G+\Delta G \\
L_{\text {var }}\left(w_{1}, w_{2}\right)=L_{0}+\Delta L_{1} w_{1}+\Delta L_{2} w_{2}=L+\Delta L
\end{gathered}
$$

To alleviate the problem of large computational complexity, the effect of process variations must be taken into account in model-order reduction algorithms. Furthermore, the resulting variational reduced-order model needs to converge to the reduced-order model of the nominal network when all the parameter variations are zero.

To obtain a balanced truncation technique that takes the process variations into account, we should first find the new system matrix $\mathbf{A}_{\text {var }}$ (the so called perturbed system matrix) of coupled interconnects affected by process variation with respect to the ideal system matrix, $\mathbf{A}$, and the perturbed susceptance and conductance matrices $\Delta L$ and $\Delta G$. Lemma 1 helps us obtain the relationship among the perturbed system matrix, the original system matrix, and the perturbed susceptance and conductance matrices. 
Lemma 1. Given an LTI system whose state-space representation is characterized by equations (1) and (2), let the susceptance and conductance matrices, $L$ and $G$, vary according to equations (30) and (31). If $\|\Delta L\|_{2}<<L \|_{2}$, then:

$$
\mathbf{A}_{v a r} \approx \mathbf{A}+\Delta \mathbf{A}
$$

where

$$
\Delta \mathbf{A}=\Delta \mathbf{A}_{1}+\Delta \mathbf{A}_{2}
$$

$$
\Delta \mathbf{A}_{1}=-L_{s 0}^{-1} \Delta G+L_{s 0}^{-1} \Delta L_{s 0} L_{s 0}^{-1} G \quad ; \quad \Delta \mathbf{A}_{2}=L_{s 0}^{-1} \Delta L_{s 0} L_{s 0}^{-1} \Delta G
$$

where $\Delta\left(L_{s 0}\right)=\Delta\left(L+G / s_{0}\right)$, and $s_{0}$ is an arbitrary, but fixed expansion point such that $\mathbf{A}=-\left[\left(L+G / s_{0}\right)^{-1} G\right]$ becomes non-singular.

Proof: Starting with the state-space representation of the perturbed system, the perturbed system matrix, $\mathbf{A}_{v a r}$ is derived by substituting (30) and (31) in the closed-form expression of the system matrix, i.e.,:

$$
\mathbf{A}_{v a r}=-\left(\mathbf{I}+L_{s 0}^{-1} \Delta L_{s 0}\right)^{-1} L_{s 0}^{-1}(G+\Delta G)
$$

Expanding the Laurent series of $\left(\mathbf{I}+L_{s 0}^{-1} \Delta L_{s 0}\right)$, and neglecting higher order terms $\left(L_{s 0}^{-1} \Delta L_{s 0}\right)^{k}$ for $k \geq 2$ directly yields the desired equation.

Lemma 1 enables one to obtain the relationship between the perturbed system matrix and the original one as well as the incremental variations of the susceptance and conductance matrices.

The balanced realization approach directly utilizes the balancing transformation to project the existing system to a new system whose controllability and observability grammians are identical and diagonalized. The diagonal elements readily represent all the singular values corresponding to the state variables of the system. The $k$-th order truncated balanced realization is then obtained by considering the $k$ largest singular-values. This approach provides insightful information about the energy exerted by each state variable, and thus, the contribution of each state variable on the external behavior of the system. From a mathematical viewpoint, the Hankel singular values of the system transfer function are indeed the eigenvalues of the symmetric matrix, $\mathbf{P}_{L} \mathbf{Q}_{L}$ of the passivity guaranteed system. To realize the effect of interconnect parameter variations on the observability and controllability grammians of the system, the following Theorem is introduced. 
Theorem 1. Consider a passive LTI system with the state-space representation given by equations (1) and (2). Suppose that the system matrix $\mathbf{A} \in \mathfrak{R}^{n \times n}$ is perturbed by $\Delta \mathbf{A} \in \mathfrak{R}^{n \times n}$. The controllability and observability grammians of the perturbed system are approximately equal to:

$$
\begin{gathered}
\mathbf{P}_{\text {var }} \cong \Delta \boldsymbol{\Upsilon} \mathbf{P}_{L} \Delta \boldsymbol{\Upsilon}^{T} \\
\mathbf{Q}_{\text {var }} \cong \Delta \boldsymbol{\Upsilon}^{T} \mathbf{Q}_{L} \Delta \boldsymbol{\Upsilon}
\end{gathered}
$$

where

$$
\Delta \mathrm{Y}=\mathbf{I}+(n-1) \cdot \Delta \mathbf{A} \cdot \mathbf{A}^{-1}
$$

Proof: A complete proof is provided for Eq. (35). Eq. (36) can be proven using a similar approach. The positive-definite controllability grammian $\mathbf{P}_{L}$ is the solution to Lur'e equation given by equations (5) and (6). Due to the similarity between the Lur'e equation and the classical Lyapunov equation used in the conventional balanced truncation method, $\mathbf{P}_{L}$ will thus have the same mathematical form as the solution to the corresponding Lyapunov equation, i.e.,

$$
\mathbf{P}_{L}=\int_{0}^{\infty} e^{\mathbf{A} t} \mathbf{K}_{c} \mathbf{K}_{c}^{T} e^{\mathbf{A}^{T} t} d t
$$

The matrix exponential $e^{\mathbf{A} t}$ can be expressed in terms of its matrix-valued power series using Cayley-Hamilton theorem [28]. In fact, according to the Cayley-Hamilton theorem, there exist analytical scalar functions, $\left.\alpha_{k}(t)\right\}_{k=0}^{n-1}$, such that:

$$
e^{\mathbf{A} t}=\sum_{k=0}^{n-1} \alpha_{k}(t) \mathbf{A}^{k}
$$

Replacing the exponential function and its transpose in Eq. (37) with their corresponding equivalent finite-series representations in Eq. (38) yields the following expression:

$$
\mathbf{P}_{L}=\int_{0}^{\infty}\left(\sum_{k=0}^{n-1} \alpha_{k}(t) \mathbf{A}^{k} \mathbf{B}\right)\left(\sum_{l=0}^{n-1} \alpha_{l}(t) \mathbf{A}^{l} \mathbf{B}\right)^{T} d t
$$

Replacing $\mathbf{A}$ in the above equation with $\mathbf{A}+\Delta \mathbf{A}$, and performing matrix factorizations will prove the validity of Eq. (35). It can also be proven that Eqs. (35) and (36) will indeed provide upper bounds for $\mathbf{P}_{v a r}$ and $\mathbf{Q}_{\text {var }}$ matrix values.

According to Theorem 1, any perturbation in the system matrix manifests itself as a congruence transformation, $\Delta \mathrm{\Upsilon}$, that maps the observability and controllability grammians of the system to the ones for the new perturbed system demonstrated by Eqs. (35) and (36). Moreover, having 
positive-definite $\mathbf{P}_{L}$ and $\mathbf{Q}_{L}$ automatically results in positive-definite $\mathbf{P}_{v a r}$ and $\mathbf{Q}_{v a r}$, because the latter matrices are congruent to the former ones and the positive-realness is retained by congruent transformations (the proof is straightforward).

To account for the effect of process variations in the proposed model-order reduction technique, Theorem 1 is directly utilized in the new algorithm. Moreover, the proposed technique also reshapes the estimated error in the frequency domain using the frequency weighting method described in Section 3, and thus, creates a reduced-order system with more accurate time- and frequency-domain responses than a Krylov-based order reduction technique. Reshaping the spectrum of the estimated error is undertaken by introducing input and/or output weighting functions that appropriately minimize(s) the error in the regions where there is a large discrepancy between the spectrum of the original system and that of reduced system. Comparing the proposed algorithm with the work in [19] on the variational Krylov-subspace model-reduction, the key advantages of variational truncated balanced realization is a superior accuracy and a proven errorbound. Under specific assumptions set forth by the perturbation theory [20], one can theoretically calculate the projection matrix of the perturbed system. Nevertheless, as also pointed out in [19], it is impractical to use the perturbation theory. Instead, reference [19] seeks to calculate the firstorder expansion of the Krylov-subspace. More precisely, the method proposed by [19] involves the calculation of the first-order expansion of the Krylov-subspace whose projection matrix is as follows:

$$
\mathbf{X}\left(w_{1}, w_{2}\right)=\mathbf{X}_{0}+\Delta \mathbf{X}_{11} w_{1}+\Delta \mathbf{X}_{21} w_{2}+\Delta \mathbf{X}_{12} w_{1}^{2}+\Delta \mathbf{X}_{22} w_{2}^{2}
$$

where $w_{l}$ and $w_{2}$ are dimensional variations. $\Delta \mathbf{X}_{i j}$ 's are to be computed by choosing a set of sample points. A variational reduced-order model can then be constructed by inserting the resulting variational Krylov-subspace into the PRIMA equations. The calculation of $\Delta \mathbf{X}_{i j}$ 's on a limited number of sample points will lead to additional errors, which may become significant in long interconnects carrying high-frequency signals.

There are, however, some problems that need to be taken into account:

- We still need to solve the Lyapunov equations to obtain the grammians of the system. To efficiently solve the Lyapunov equations, an iterative Lyapunov equation solver, Vector ADI (VADI), which was presented in [11] and [12], is utilized. The VADI algorithm was developed to provide a low-rank approximation to the solution of the Lyapunov equations. The VADI method is as inexpensive as Krylov subspace-based moment matching methods. Reference [12] includes a discussion about the number of required iterations and an analysis of the computational complexity of the VADI method. 
- To account for the effect of process variations on the interconnect performance, Theorem 1 is directly employed. However, equations (35) and (36) involve a matrix inversion operation. To compute the inverse of a matrix more efficiently, the problem is elaborated in the form of solving a set of linear algebraic equations rather than explicit inverse formation [20].

Now, we proceed with describing the new procedure for variational balanced truncation. Given $\mathbf{H}(s), \mathbf{W}_{i}(s), \mathbf{W}_{o}(s)$ :

1. Using lemma 1, Determine matrix $\Delta \mathbf{A}$.

2. Using Theorem 1, compute the perturbing congruence transformation, $\Delta \mathrm{r}$.

3. Using the VADI iteration, compute the controllability and observability grammians of $\mathbf{P}_{L}$ and $\mathbf{Q}_{L}$ of the original spectrally-weighted system by solving the Lur'e equations (19) and (20).

4. Calculate new perturbed controllability and observability grammians, $\mathbf{P}_{v a r}$ and $\mathbf{Q}_{\text {var }}$ using Eqs. (35) and (36).

5. Using equations (23) and (24), compute $\mathbf{X}_{v a r}$ and $\mathbf{Y}_{v a r}$ from $\mathbf{P}_{v a r}$ and $\mathbf{Q}_{v a r}$.

6. Decompose $\mathbf{X}_{v a r}$ and $\mathbf{Y}_{v a r}$ using the eigenvalue decomposition technique into $\mathbf{U}_{v a r} \mathbf{S}_{v a r} \mathbf{U}_{v a r}^{T}$ and $\mathbf{V}_{v a r} \mathbf{Z}_{v a r} \mathbf{V}_{v a r}^{T}$.

7. Use equations (27) and (28), compute $\overline{\mathbf{B}}_{\text {var }}$ and $\overline{\mathbf{C}}_{v a r}$.

8. Using the VADI iteration, solve the mapped Lur'e equations (21) and (22) for the new perturbed system to compute $\hat{\mathbf{P}}_{\text {var }}$ and $\hat{\mathbf{Q}}_{\text {var }}$.

9. Using the block Lanczos algorithm, obtain the reduced order left and right eigen-matrices, $\hat{\mathbf{V}}_{L, v a r}$ and $\hat{\mathbf{V}}_{R, v a r}$, associated with the $k$ largest singular values of S $\hat{\mathbf{P}}_{v a r} \hat{\mathbf{Q}}_{v a r}$.

10. Let $\hat{\mathbf{E}}_{\text {var }}=\hat{\mathbf{V}}_{L, v a r}{ }^{T} \hat{\mathbf{V}}_{R \text {,var }}$ and compute the singular-value decomposition of $\hat{\mathbf{E}}_{\text {var }}$, i.e., $\hat{\mathbf{E}}_{\text {var }}=\hat{\mathbf{U}}_{E, v a r} \hat{\Sigma}_{E, v a r} \hat{\mathbf{V}}_{E, v a r}^{T}$.

11. Let $\hat{\mathbf{S}}_{L, v a r}=\hat{\mathbf{V}}_{L, v a r} \hat{\mathbf{U}}_{E, v a r} \hat{\Sigma}_{E, v a r}^{-1 / 2} \in \Re^{n \times k}$ and $\quad \hat{\mathbf{S}}_{R, v a r}=\hat{\mathbf{V}}_{R, v a r} \hat{\mathbf{V}}_{E, v a r} \hat{\Sigma}_{E, v a r}^{-1 / 2} \mathfrak{R}^{n \times k}$

12. Compute the reduced order space realization using the matrices $\widehat{\mathbf{S}}_{L, v a r}$ and $\widehat{\mathbf{S}}_{R, \text { var }}$ as follows: 


$$
\left[\begin{array}{c|c}
\hat{\mathbf{A}}_{v a r} & \hat{\mathbf{B}}_{v a r} \\
\hline \hat{\mathbf{C}}_{v a r} & \hat{\mathbf{D}}_{v a r}
\end{array}\right]=\left[\begin{array}{c|c}
\hat{\mathbf{S}}_{L, v a r} \mathbf{A}_{v a r} \hat{\mathbf{S}}_{R, v a r} & \hat{\mathbf{S}}_{L, v a r}^{T} \mathbf{B} \\
\hline \mathbf{C S}_{R, v a r} & \mathbf{D}
\end{array}\right]
$$

Notice that to guarantee the passivity, the Lur'e equations must be solved instead of Lyapunov equations, leading to a slight modification of the above algorithm.

Now we prove that a provable error bound exists on the $L^{\infty}$-norm of the weighted error between the original system and the reduced order system obtained using the proposed variational, spectrally-weighted balanced truncation.

Theorem 2. The $L^{\infty}$-error of the variational model reduction with spectral shaping is:

$$
\left\|\mathbf{W}_{o}(s)\left(\mathbf{H}_{v a r}(s)-\mathbf{H}_{r_{v a r}}^{k}(s)\right) \mathbf{W}_{i}(s)\right\|_{\infty} \leq k\left(\sum_{i=k+1}^{n} \sigma_{v a r, i}\right)
$$

where $\sigma_{v a r, i}(i=1, \ldots, n)$ are singular values of the perturbed system. $k=\left\|\mathbf{W}_{o}(s) \mathbf{L}\right\|_{\infty}\left\|\mathbf{K} \mathbf{W}_{i}(s)\right\|_{\infty}$ and

$$
\begin{gathered}
\mathbf{K}=\operatorname{diag}\left(\left|s_{1}\right|^{-1 / 2}, \ldots,\left|s_{n}\right|^{-1 / 2}, 0, \ldots, 0\right) \mathbf{U}^{T} \mathbf{B} \\
\mathbf{L}=\mathbf{C V} \operatorname{diag}\left(\left|z_{1}\right|^{-1 / 2}, \ldots,\left|z_{n}\right|^{-1 / 2}, 0, \ldots, 0\right)
\end{gathered}
$$

Proof: Expanding the left-hand side of Eq. (13) in terms of Laplace-domain expressions of $\mathbf{H}(s)$ and $\mathbf{H}_{r_{v a r}}^{k}(s)$ results in the following equation:

$$
\left\|\mathbf{W}_{o}(s)\left(\mathbf{C}\left(s \mathbf{I}-\mathbf{A}_{v a r}\right)^{-1} \mathbf{B}-\hat{\mathbf{C}}_{v a r}\left(s \mathbf{I}-\hat{\mathbf{A}}_{v a r}\right)^{-1} \hat{\mathbf{B}}_{v a r}\right) \mathbf{W}_{i}(s)\right\|_{\infty}
$$

In the spectrally-weighted variational balanced truncation, the reduced-order system is derived by applying the transformation matrix, $\mathbf{T}_{v a r}$, on the original perturbed system and then truncating the transformed system as mathematically described by equations (8)-(10):

$$
\left[\begin{array}{c|c}
\mathbf{T}_{\text {var }} \mathbf{A}_{\text {var }} \mathbf{T}_{\text {var }}^{-1} & \mathbf{T B} \\
\hline \mathbf{C T}^{-1} & \mathbf{D}
\end{array}\right]=\left[\begin{array}{cc|c}
\hat{\mathbf{A}}_{\text {var }} & \mathbf{A}_{12} & \hat{\mathbf{B}}_{\text {var }} \\
\mathbf{A}_{21} & \mathbf{A}_{22} & \mathbf{B}_{2} \\
\hline \hat{\mathbf{C}}_{\text {var }} & \mathbf{C}_{2} & \mathbf{D}
\end{array}\right]
$$


Matrices $\overline{\mathbf{B}}_{\text {var }}$ and $\overline{\mathbf{C}}_{\text {var }}$, obtained from the seventh step of the spectrally-weighted variational balanced truncation algorithm, are also partitioned correspondingly. It is readily proved that $\hat{\mathbf{B}}_{v a r}=\overline{\mathbf{B}}_{v a r, 1} \mathbf{K}$ and $\hat{\mathbf{C}}_{v a r}=\mathbf{L} \overline{\mathbf{C}}_{v a r, 1}$. Thus:

$$
\begin{aligned}
& \left\|\mathbf{W}_{o}(s)\left(\mathbf{C}\left(s \mathbf{I}-\mathbf{A}_{v a r}\right)^{-1} \mathbf{B}-\hat{\mathbf{C}}_{v a r}\left(s \mathbf{I}-\hat{\mathbf{A}}_{v a r}\right)^{-1} \hat{\mathbf{B}}_{v a r}\right) \mathbf{W}_{i}(s)\right\|_{\infty}= \\
& \left.\| \mathbf{W}_{o}(s)\left(\mathbf{L} \overline{\mathbf{C}}_{v a r}\left(s \mathbf{I}-\mathbf{A}_{v a r}\right)^{-1} \overline{\mathbf{B}}_{v a r} \mathbf{K}\right)-\mathbf{L} \overline{\mathbf{C}}_{v a r, 1}\left(s \mathbf{I}-\hat{\mathbf{A}}_{v a r}\right)^{-1} \overline{\mathbf{B}}_{v a r, 1} \mathbf{K}\right) \mathbf{W}_{i}(s) \|_{\infty}= \\
& \left.\| \mathbf{W}_{o}(s) \mathbf{L}\left(\overline{\mathbf{C}}_{v a r}\left(s \mathbf{I}-\mathbf{A}_{v a r}\right)^{-1} \overline{\mathbf{B}}_{v a r}\right)-\overline{\mathbf{C}}_{v a r, 1}\left(s \mathbf{I}-\hat{\mathbf{A}}_{v a r}\right)^{-1} \overline{\mathbf{B}}_{v a r, 1}\right) \mathbf{K} \mathbf{W}_{i}(s) \|_{\infty} \leq \\
& \left.\left\|\mathbf{W}_{o}(s) \mathbf{L}\right\|_{\infty} \times \| \overline{\mathbf{C}}_{v a r}\left(s \mathbf{I}-\mathbf{A}_{v a r}\right)^{-1} \overline{\mathbf{B}}_{v a r}-\overline{\mathbf{C}}_{v a r, 1}\left(s \mathbf{I}-\hat{\mathbf{A}}_{v a r}\right)^{-1} \overline{\mathbf{B}}_{v a r, 1}\right)\left\|_{\infty} \times\right\| \mathbf{K} \mathbf{W}_{i}(s) \|_{\infty}
\end{aligned}
$$

Note that $\overline{\mathbf{C}}_{v a r, 1}\left(s \mathbf{I}-\hat{\mathbf{A}}_{v a r}\right)^{-1} \overline{\mathbf{B}}_{v a r, 1}$ represents the reduced-order model of $\overline{\mathbf{C}}_{v a r}\left(s \mathbf{I}-\mathbf{A}_{v a r}\right)^{-1} \overline{\mathbf{B}}_{v a r}$. Hence, there exists a bounded $L^{\infty}$-error for the former system whose reduced-order model is represented by the latter system.

$$
\frac{1}{2}\left\|\left(\overline{\mathbf{C}}_{v a r}\left(s \mathbf{I}-\mathbf{A}_{v a r}\right)^{-1} \overline{\mathbf{B}}_{v a r}-\overline{\mathbf{C}}_{v a r, 1}\left(s \mathbf{I}-\hat{\mathbf{A}}_{v a r}\right)^{-1} \overline{\mathbf{B}}_{v a r, 1}\right)\right\|_{\infty} \leq \sum_{i=k+1}^{n} \sigma_{v a r, i}
$$

Plugging the above equation into Eq. (41) proves the Theorem.

As mentioned earlier, process variations will have a definite impact on the on-chip interconnect parasitics. These variations have two adverse effects on the system matrix as also demonstrated by Eqs. (32)-(33). Ignoring second-order variations, any incremental increase in the values of parasitic conductances and resistances reduces $\Delta \mathbf{A}$, whereas any incremental increase in the values of parasitic inductances and capacitances increases $\Delta \mathbf{A}$.

As an interesting special case, consider the tightly coupled RLC interconnects shown in Fig. 3. It is easily proved that, for this case, the system matrix $\mathbf{A}$ is a symmetric positive-definite matrix (the susceptance and conductance matrices are both symmetric positive definite matrices). Under these circumstances, the following theorem proves useful in finding the upper and lower limits of variations in the poles of the system transfer function of tightly coupled RLC interconnects that are subject to the process variations: 


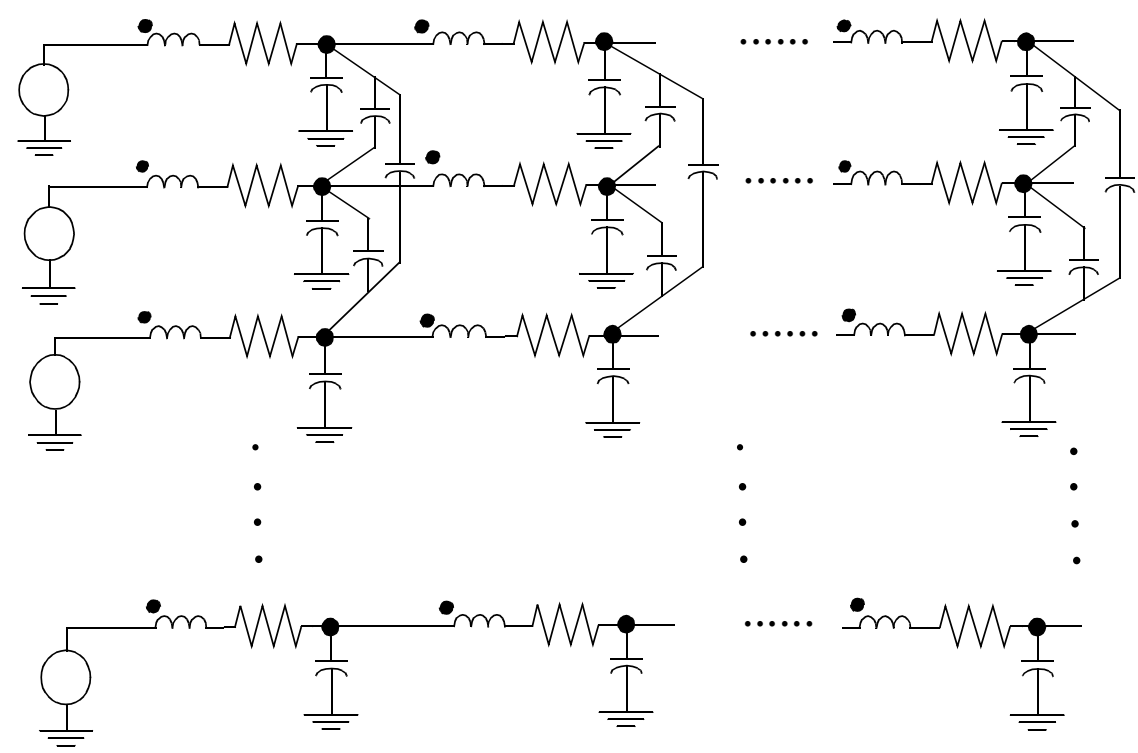

Fig. 3. Circuit schematic of $\mathrm{N}$ interconnects that are electromagnetically coupled to each other

Theorem 3 [20]. Given an LTI system whose state-space representation is given by equations (1) and (2), if the system matrix $\mathbf{A} \in \mathfrak{R}^{n \times n}$ is perturbed by $\Delta \mathbf{A} \in \mathfrak{R}^{n \times n}$ due to the process variation, then the following inequalities hold:

$$
\begin{gathered}
\left|\lambda_{k}(\mathbf{A}+\Delta \mathbf{A})-\lambda_{k}(\mathbf{A})\right| \leq\|\Delta \mathbf{A}\|_{2} \\
\lambda_{k}(\mathbf{A})+\lambda_{n}(\Delta \mathbf{A}) \leq \lambda_{k}(\mathbf{A}+\Delta \mathbf{A}) \leq \lambda_{k}(\mathbf{A})+\lambda_{1}(\Delta \mathbf{A})
\end{gathered}
$$

where $\Delta \mathbf{A}$ is given by lemma 1 .

Proof hint: Follows from theories developed for the symmetric eigenvalue problem (see pages 395-400 of [20]).

According to Theorem 3, the magnitude of difference between poles of the perturbed system and those of the original system is limited by the $\mathrm{L}_{2}$-norm of the perturbing matrix, $\Delta \mathbf{A}$. Furthermore, the eigenvalues of the perturbed system are upper and lower bounded by the values given in Eq. (43).

\section{Experimental results}

In this section the proposed variational spectrally-weighted balanced truncation model-order reduction technique (VSBT) is evaluated by performing experiments on a number of global interconnect structures such as clock trees and coupled interconnect lines. To preserve passivity, the proposed VSBT incorporates the algorithm proposed in [13]. 
First, the accuracy of spectrally-weighted balanced truncation (SBT) technique [16]-[18] is demonstrated and the result of applying this algorithm is compared with those obtained by utilizing our implementations of PRIMA [6] and the positive-real truncated balanced realization (PRTBR) [13]. The VSBT method is then applied to study the impact of the interconnect process variations on the timing performance of the clock trees and coupled busses. Finally, the accuracy of VSBT is validated by using it to reduce the order of an arbitrary stable LTI system that is subject to perturbations.

\subsection{A single lossy distributed RLC interconnect}

First, consider a single lossy transmission line of 3-mm length. The values for unit-length capacitances, inductances and resistances are demonstrated in Fig. 4. The transmission line is modeled by 1000 lumped RLC sections in cascade, hence, the system has an order of 2000. Shown in Fig. 5 is the magnitude response of the reduced transfer function obtained by the proposed technique compared to those of the PR-TBR algorithm and PRIMA algorithm. The order of the reduced systems is set to four. The input weighting function is identified as a lead compensator with its zero located at a lower frequency compared to its pole location, i.e.,

$$
W_{i}(s)=\frac{8 \times 10^{8}\left(s+10^{7}\right)}{10^{7}\left(s+8 \times 10^{8}\right)}
$$

Clearly, the SBT technique produces a reduced system with a frequency response closely following that of the original system. The weighting function in Eq. (44) is capable of maintaining the low-frequency accuracy, while significantly improving the high-frequency accuracy up to $10 \mathrm{GHz}$.

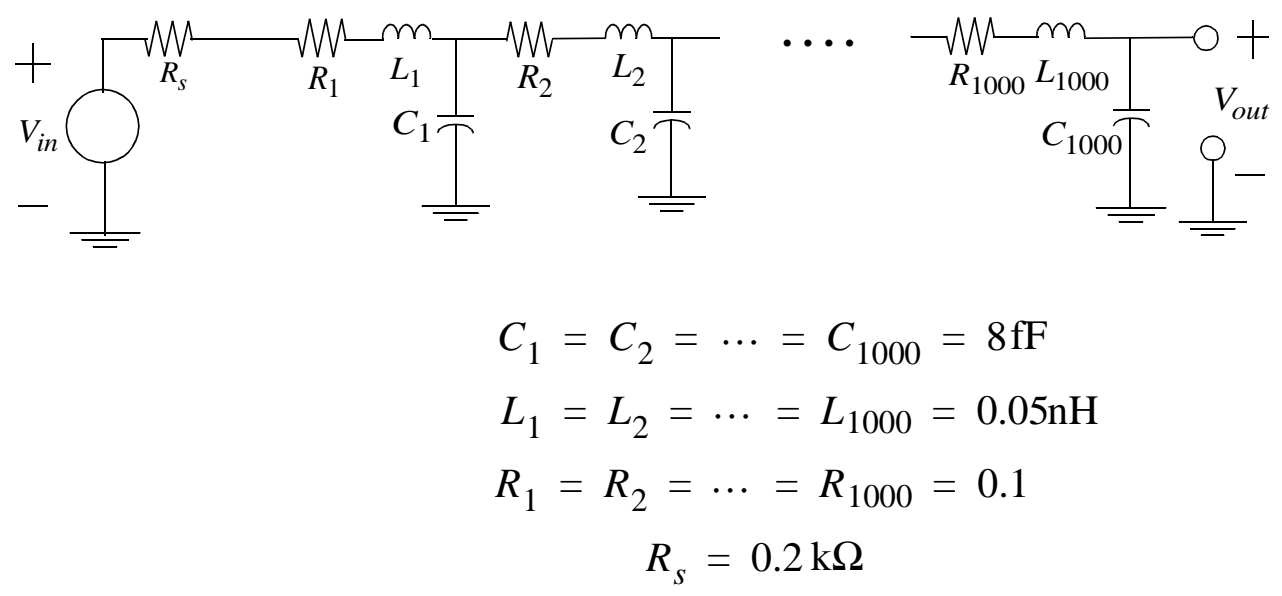

Fig. 4. A single lossy line modeled by 1000 ladder RLC sections 


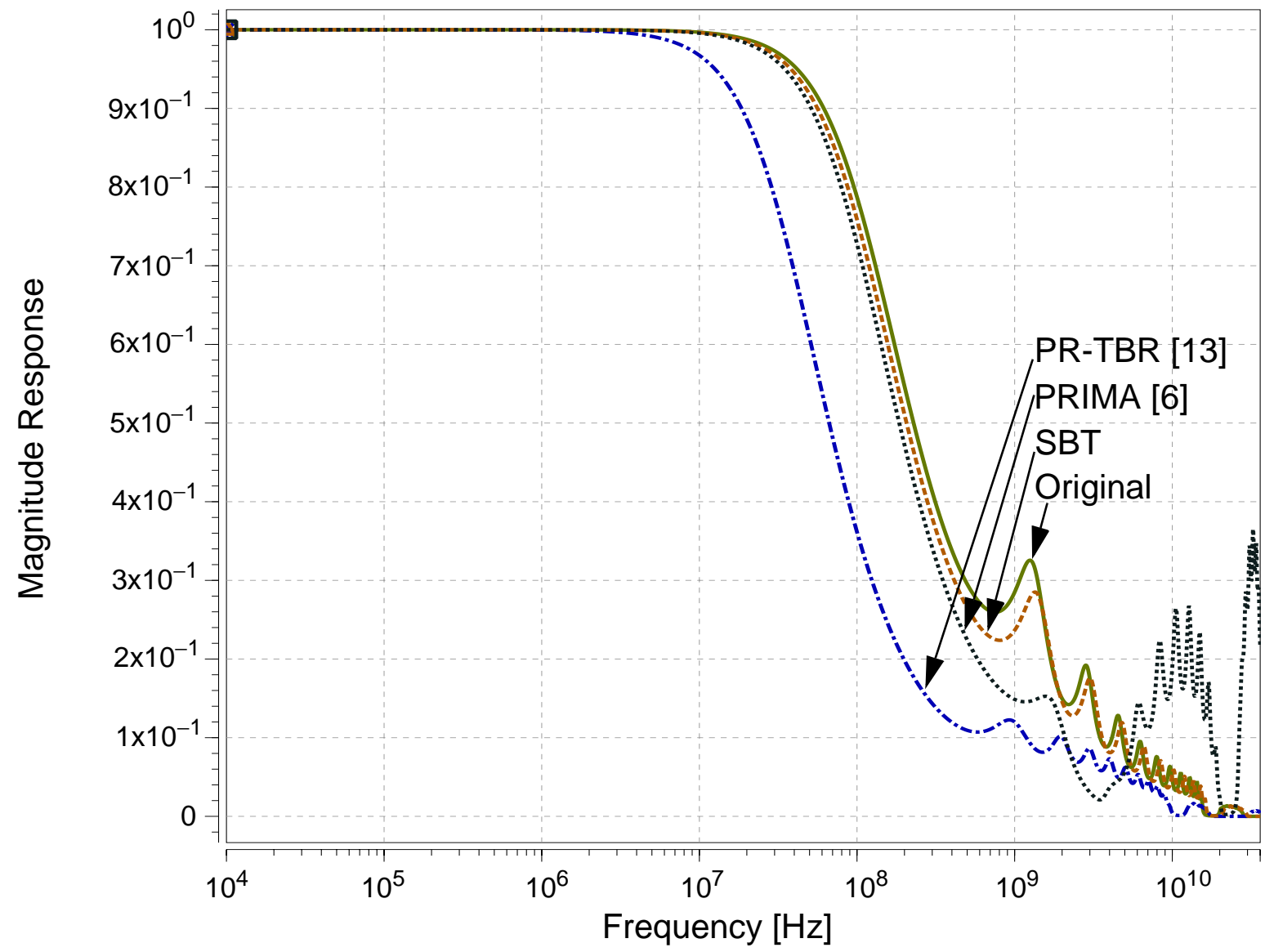

Fig. 5. The Bode diagram of a single interconnect having 1000 lumped RLC sections and the corresponding reduced-order systems

\subsection{Two electromagnetically-coupled interconnects}

To examine the performance of the proposed SBT technique in high-frequency coupled interconnects, two electromagnetically-coupled interconnects are considered. Shown in Fig. 6 is the distributed RLC model for these two interconnects. The values for the electrical elements of interconnects are also indicated underneath the circuit schematic. The capacitive coupling is modeled by distributed floating capacitances, $C_{c}$. In state-of-the-art CMOS technologies, the coupling capacitances account for approximately $70-95 \%$ of the total node capacitances, which makes the coupling noise analysis even more important. Similarly, mutual inductances with a coupling coefficient of $k$ capture the inductive coupling between the two interconnects. To include the most general case, the electrical values of the two lines are considered to be different. $\mathrm{C}_{\mathrm{L} 1}$ and $\mathrm{C}_{\mathrm{L} 2}$ represent the load capacitances at the far end terminations of the aggressor and victim lines. In highspeed ICs, the lines are normally driving other CMOS buffers, therefore, the load capacitances are

comprised of the input capacitances of the CMOS receiver buffers driven by the interconnects. 
The receiver buffer sizes for long interconnects are rather large, leading to large load capacitances, $\mathrm{C}_{\mathrm{L} 1}$ and $\mathrm{C}_{\mathrm{L} 2}$. Each line is modeled using 500 ladder RLC sections in cascade, to accurately capture the distributed nature of long interconnects in high-speed VLSI circuits.

In this experiment, the accuracy of the proposed SBT method in estimating the spectrum of electromagnetic crosstalk is examined. The frequency response of the two coupled RLC interconnects with the input signal $V_{\text {in }}$ at the near end of the aggressor line and the output voltage across the load capacitance $\mathrm{C}_{\mathrm{L} 2}$ at the far end termination of the victim line is first simulated using HSPICE. Next, three model-order reduction techniques are employed to reduce the order of the original system: PRIMA [6], PR-TBR [13], and the proposed SBT method. The order of the system is reduced to eight using these three different order-reduction techniques. For the SBT method, a first-order lowpass weighting function, $W_{i}(s)=1 /\left(1+s \tau_{0, w i}\right)$, at the input is incorporated. To calculate the time-constant of the weighting function, $\tau_{0, w i}$, an important attribute of the crosstalk noise is utilized. The time-domain crosstalk voltage experiences its maximum gradient around its $50 \%$ rising transition time. The $50 \%$ rising transition time is calculated by using an extension to the Elmore delay metric for RLC tree circuits [28]. The maximum time-domain variation corresponds to the high-frequency spectral components of the signal. As a consequence, an input weighting function with a time-constant equal to $50 \%$ of the rising transition time of the far end crosstalk greatly reduces the high-frequency imprecision of the low-order reduced system obtained by PRIMA and PR-TBR techniques. This is evident from Fig. 7 where the magnitude response of the two coupled RLC circuits in Fig. 6 is compared with those of the reduced systems. 


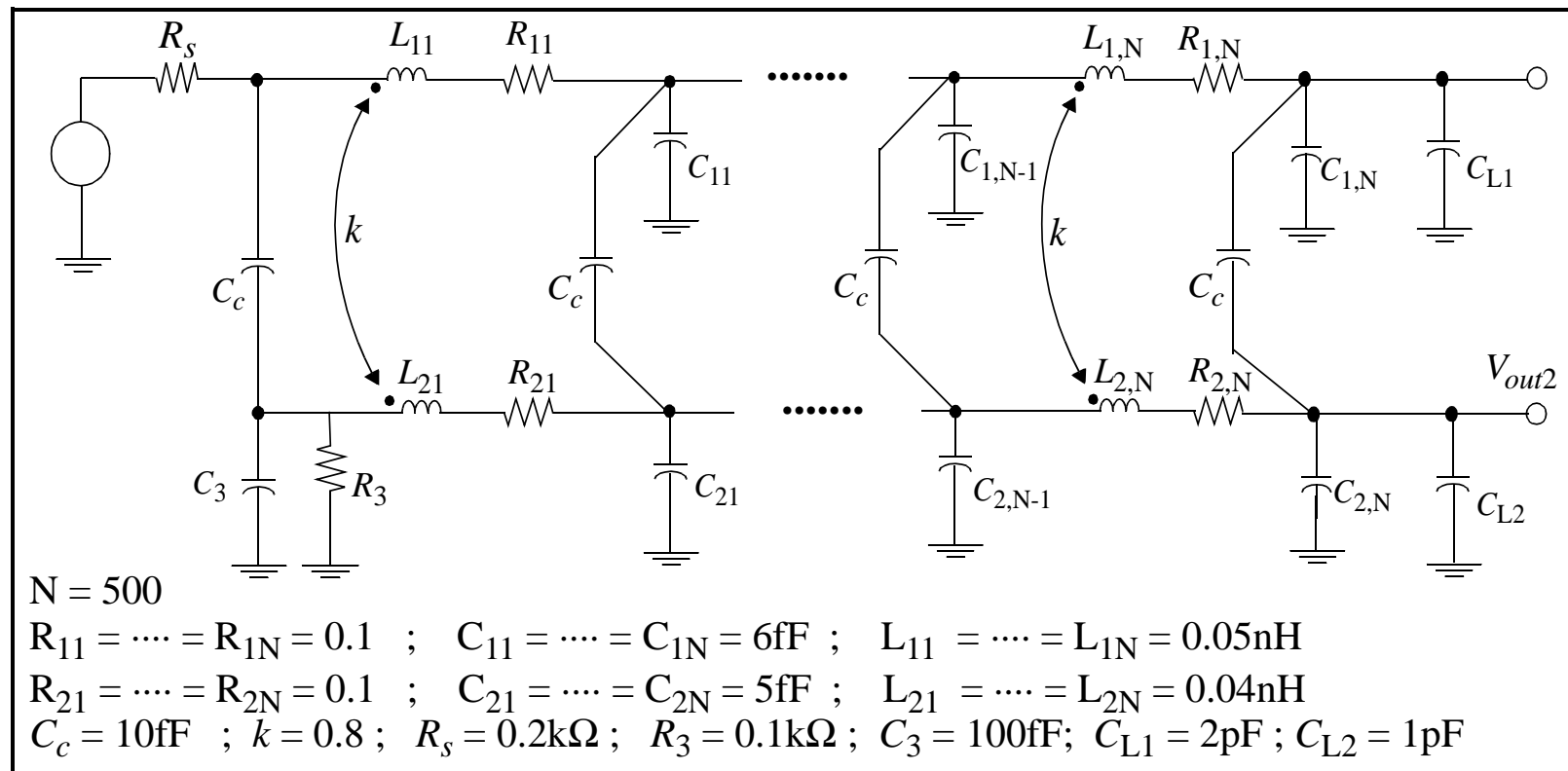

Fig. 6. Two electromagnetically coupled RLC interconnects.

All of the order reduction techniques in this experiment accurately predict the spectrum of the far end crosstalk at the low frequency range. However, PRIMA and PR-TBR are incapable of following the signal spectrum for frequencies beyond $700 \mathrm{MHz}$. In contrast, SBT follows the spectral variations of the crosstalk for frequencies up to $7 \mathrm{GHz}$.

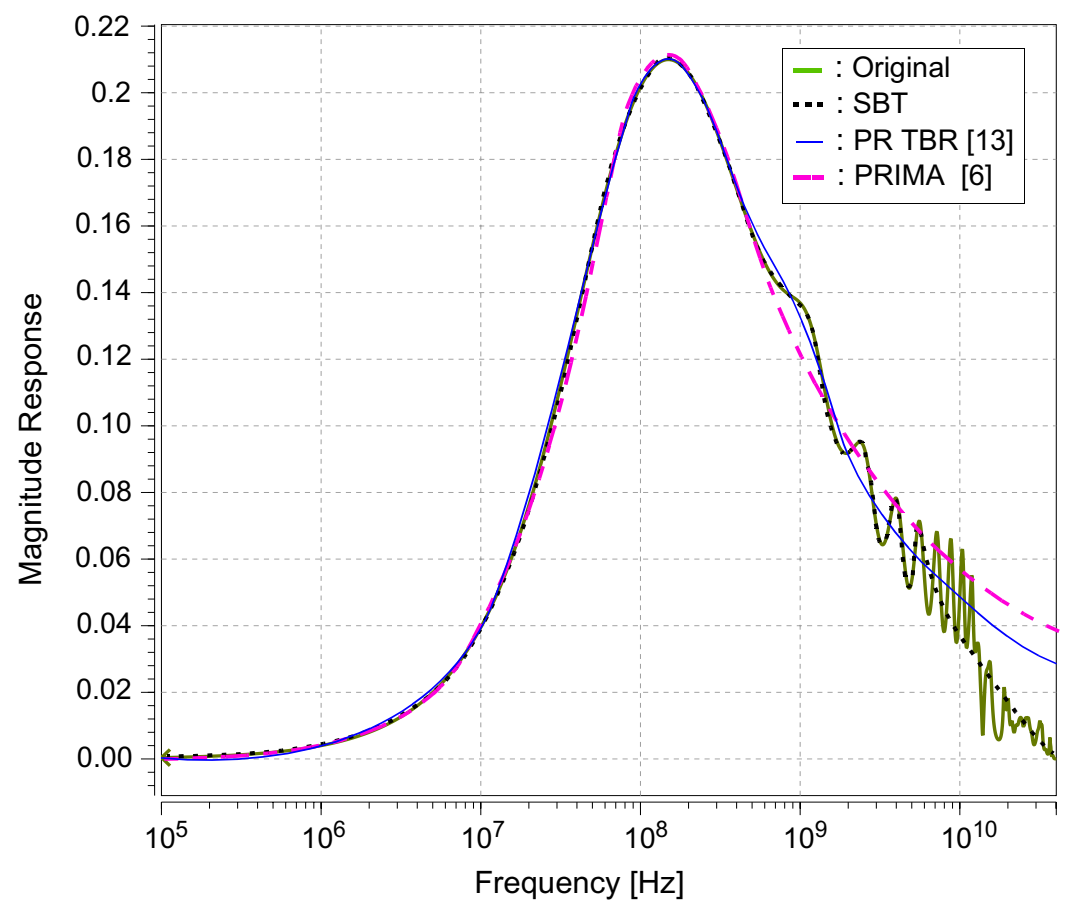

Fig. 7. The magnitude response of the original system and reduced-order systems obtained by using SPBT, PR-TBR[13], PRIMA [16]. 


\subsection{H-tree clock distribution}

An H-tree clock network is constructed and routed in the TSMC $0.13 \mu \mathrm{m}$ digital CMOS technology. The clock tree is driven by a four-stage tapered buffer at the root of the tree, as shown in Fig. 8. The design target of the on-chip clock frequency is $3.0 \mathrm{GHz}$. The clock tree is modeled by a large coupled distributed RLC network. More precisely, every $5 \mu \mathrm{m}$ segment of each line is modeled by an RLC ladder network, whose R, L, and C values are changed due to metal width and ILD thickness variations. Based on the data reported in [26] on the interconnect-dominated test circuits, the typical variational distribution for metal interconnects as well as ILD thicknesses is a normal distribution. The widths of metal and ILD layers vary up to $30 \%$ of their nominal values (which is the ratio of the $3-\sigma$ variation to the nominal value stated as a percentage).

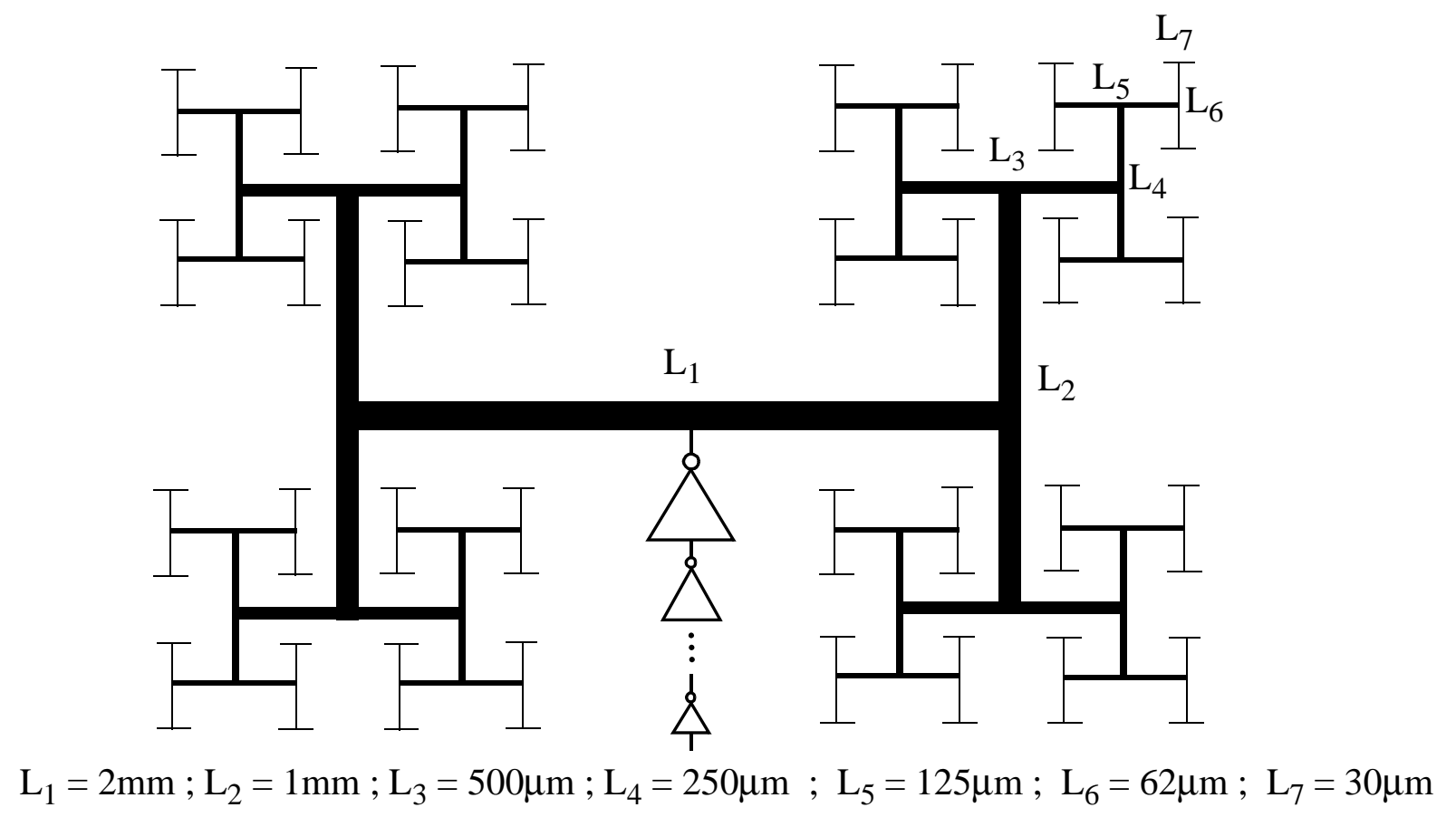

Fig. 8. The H-tree clock distribution driven by a tapered buffer 
Table 1: Comparison between simulation results on an H-tree clock net with process variations (Star-HSPICE level 49, 0.13 $\mu \mathrm{m}$, CMOS process). Delays are given in ps.

\begin{tabular}{|c|c|c|c|c|}
\hline \multicolumn{2}{|c|}{$\begin{array}{c}3-\sigma \text { variations } \\
(\%)\end{array}$} & \multicolumn{3}{|c|}{$\begin{array}{c}50 \% \text { delay } \\
\text { of the fan-out node }\end{array}$} \\
\hline ILD width & $\begin{array}{l}\text { Metal Layer } \\
\text { width }\end{array}$ & $\begin{array}{l}\text { paper [19] } \\
\text { (ps) }\end{array}$ & $\begin{array}{l}\text { VSBT } \\
\text { (ps) }\end{array}$ & $\begin{array}{l}\text { Star-HSPICE } \\
(\mathrm{ps})\end{array}$ \\
\hline 12.3 & -10.3 & 195.7 & 196.0 & 196.3 \\
\hline 5.8 & 19.8 & 211.5 & 216.4 & 216.2 \\
\hline-21.4 & -2.3 & 192.3 & 200.1 & 200.1 \\
\hline-10.2 & -8.2 & 201.0 & 208.2 & 206.6 \\
\hline 2.45 & 22.1 & 200.4 & 204.1 & 203.2 \\
\hline 19.2 & 10.6 & 190.1 & 195.0 & 194.2 \\
\hline-24.3 & -17.8 & 198.2 & 200.3 & 200.0 \\
\hline 28.1 & 11.4 & 212.3 & 222.5 & 221.7 \\
\hline-12.4 & -5.9 & 214.5 & 221.9 & 220.9 \\
\hline-14.9 & -2.5 & 184.1 & 196.2 & 195.6 \\
\hline-1.3 & 12.6 & 203.6 & 213.2 & 212.5 \\
\hline 25.7 & -7.2 & 202.2 & 207.3 & 206.4 \\
\hline 7.6 & 1.6 & 202.7 & 217.7 & 215.3 \\
\hline-2.3 & -29.1 & 212.2 & 233.4 & 231.8 \\
\hline 20.1 & -18.5 & 198.4 & 211.8 & 210.5 \\
\hline-18.8 & 13.7 & 182.2 & 201.0 & 200.3 \\
\hline 22.4 & -4.5 & 186.0 & 204.7 & 203.3 \\
\hline-27.6 & 13.6 & 181.7 & 193.1 & 192.8 \\
\hline 5.3 & 14.3 & 208.5 & 218.2 & 217.8 \\
\hline 28.7 & -26.4 & 213.2 & 235.2 & 238.8 \\
\hline-13.4 & -10.6 & 185.5 & 198.1 & 197.5 \\
\hline
\end{tabular}

Twenty experiments were carried out where, in each experiment, a set of normally-distributed numbers for the metal and ILD width variations were generated. The $50 \%$ propagation delay at an arbitrarily chosen fan-out (leaf) node was computed by the VSBT technique and compared with the result obtained using the algorithm presented in [19]. Results of this comparison are provided in Table 1. Throughout this experiment, for the sake of simplicity, the geometrical variations of the interconnect are assumed to be mutually independent. In all of these experiments, the following input weighting function was utilized to minimize the high-frequency components of the estimated error: 


$$
W_{i}(s)=\frac{4 \times 10^{7}}{s+\left(4 \times 10^{7}\right)}
$$

From Table 1, it is clear that the VSBT algorithm can predict the 50\% delays more accurately than [19] for all possible 3- $\sigma$ variations of metal and ILD layers used in Table 1. By comparing the number of floating point operation (flops) from MATLAB simulation, the computation time for the VSBT technique is, on average, $15 \%$ lower than that of [19]. Notice that although the computation time for VSBT is not that much faster than that of [19], VSBT results in far more accurate delay values compared to [19].

\subsection{Two electromagnetically coupled interconnects with variational parameters}

The next experiment is on two electromagnetically-coupled microstrip lines with statistically varying electrical parameters due to process variations. Microstrip lines serve as the most appropriate models for characterizing the topmost metal layers in CMOS technology. The schematic of these two coupled lines along with their nominal geometrical parameters are depicted in Fig. 9. The accuracy of two variational order reduction techniques, the proposed VSBT and the technique proposed in [19], in estimating the delay of signal at the far end (load termination) of Line 2 is examined.

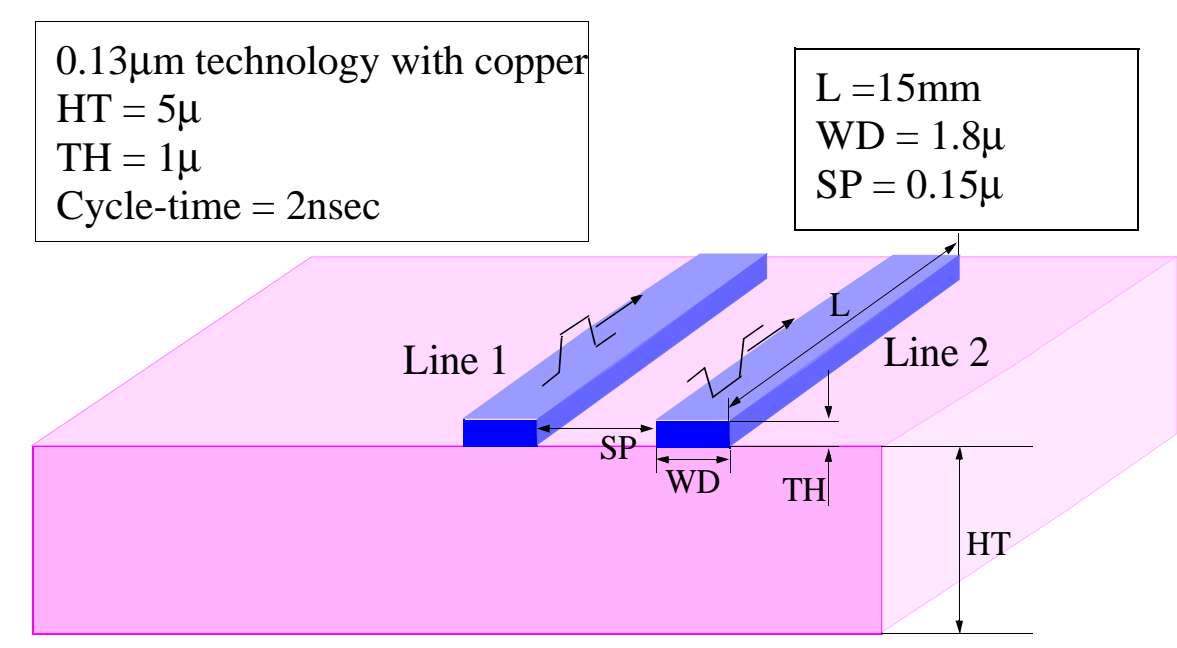

Fig. 9. Two parallel microstrip lines in a $0.13 \mu \mathrm{m}$ CMOS process

The height and width of the metal layer are subject to $\pm 30 \%$ variations around their nominal values. Twenty experiments were carried out, while allowing the metal height and width to vary as normally distributed random processes. The following input weighting function was employed to reshape the spectrum of the error: 


$$
W_{i}(s)=\frac{1 \times 10^{8}}{s+\left(1 \times 10^{8}\right)}
$$

Results of these twenty experiments are reported in Table 2. The MATLAB-reported flop usage of the VSBT technique is $10 \%$ more than that of [19], while the delays reported by VSBT is more accurate than [19].

Table 2: Comparison between simulation results on two capacitively coupled microstrip lines

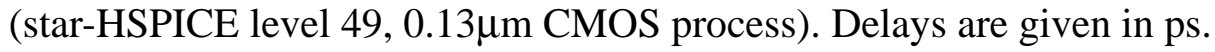

\begin{tabular}{|c|c||c|l|l|}
\hline \multicolumn{2}{|c||}{$\begin{array}{c}\text { 3- } \sigma \text { variations } \\
(\%)\end{array}$} & \multicolumn{3}{c|}{ of the far-end node of Line 2 (ps) } \\
\hline $\begin{array}{c}\text { Metal } \\
\text { W }\end{array}$ & $\begin{array}{c}\text { Metal } \\
\text { H }\end{array}$ & $\begin{array}{c}\text { Paper [19] } \\
(\mathrm{ps})\end{array}$ & $\begin{array}{c}\text { VSBT } \\
(\mathrm{ps})\end{array}$ & $\begin{array}{c}\text { Star-HSPICE } \\
(\mathrm{ps})\end{array}$ \\
\hline-0.4 & 20.1 & 598 & 597 & 596 \\
\hline 15.8 & -1.9 & 606 & 640 & 639 \\
\hline-9.3 & 13.2 & 509 & 525 & 524 \\
\hline-1.75 & 10.8 & 591 & 612 & 610 \\
\hline 15.8 & -14.4 & 680 & 700 & 697 \\
\hline-13.2 & -0.9 & 706 & 734 & 731 \\
\hline 19.1 & -29.1 & 701 & 725 & 722 \\
\hline 29.3 & 2.5 & 502 & 538 & 536 \\
\hline-1.8 & 7.9 & 583 & 610 & 607 \\
\hline 11.5 & -4.2 & 653 & 683 & 680 \\
\hline-15.3 & 19.4 & 643 & 665 & 662 \\
\hline 14.7 & 25.3 & 548 & 567 & 563 \\
\hline 1.1 & -10.4 & 643 & 667 & 665 \\
\hline-15.6 & 22.1 & 696 & 717 & 711 \\
\hline 18.2 & -23.5 & 611 & 631 & 629 \\
\hline-13.2 & -0.5 & 686 & 705 & 703 \\
\hline-9.4 & 21.7 & 629 & 644 & 643 \\
\hline 0.7 & -11.1 & 695 & 713 & 712 \\
\hline 29.8 & -20.6 & 556 & 598 & 592 \\
\hline 10.8 & -0.7 & 601 & 630 & 627 \\
\hline
\end{tabular}

\subsection{A general example}

To demonstrate the accuracy and validity of VSBT on any arbitrary stable LTI system exposed to perturbations, we started from an arbitrary state-space specification of an LTI system having twenty poles and thirteen zeros, as shown in the pole-zero map of Fig. 10. Suppose that each and 
every element of the system matrix A experiences a normally distributed random perturbation with a standard deviation of $30 \%$ around the nominal value. The order of the reduced order system is set to three. It is desired to have high accuracy in high frequencies, therefore, the following weighting function is used:

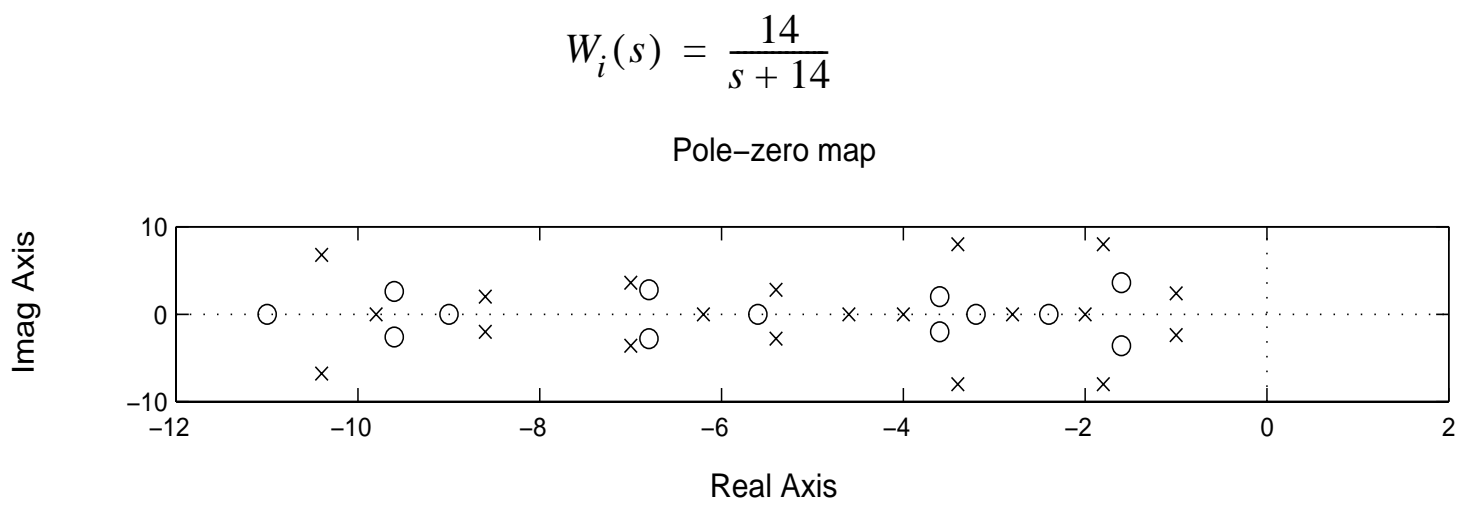

Fig. 10. The pole-zero map of the LTI system specified in section 6.5

A variational spectrally-weighted reduced-order model is derived by using two approaches. The first approach is to calculate the perturbed system matrix and then directly apply the spectrally weighted balanced truncation. The second approach is to use the VSBT technique.

Fig. 11 shows and compares the bode diagram of the magnitude response, the bode diagram of the phase response, the impulse response of the original perturbed system, the reduced-order system by using direct order reduction technique, and the reduced-order system by using the VSBT technique. Notice that applying the direct mode-order reduction technique on a large system of parameter-varying interconnects to generate the reduced order system is very time consuming. The reason for this is that the direct order reduction algorithm must be run on the system each time under different offset values for electrical parameters of the interconnect. As can be seen from Fig. 11, the difference between the two order reduction approaches is quite small. 

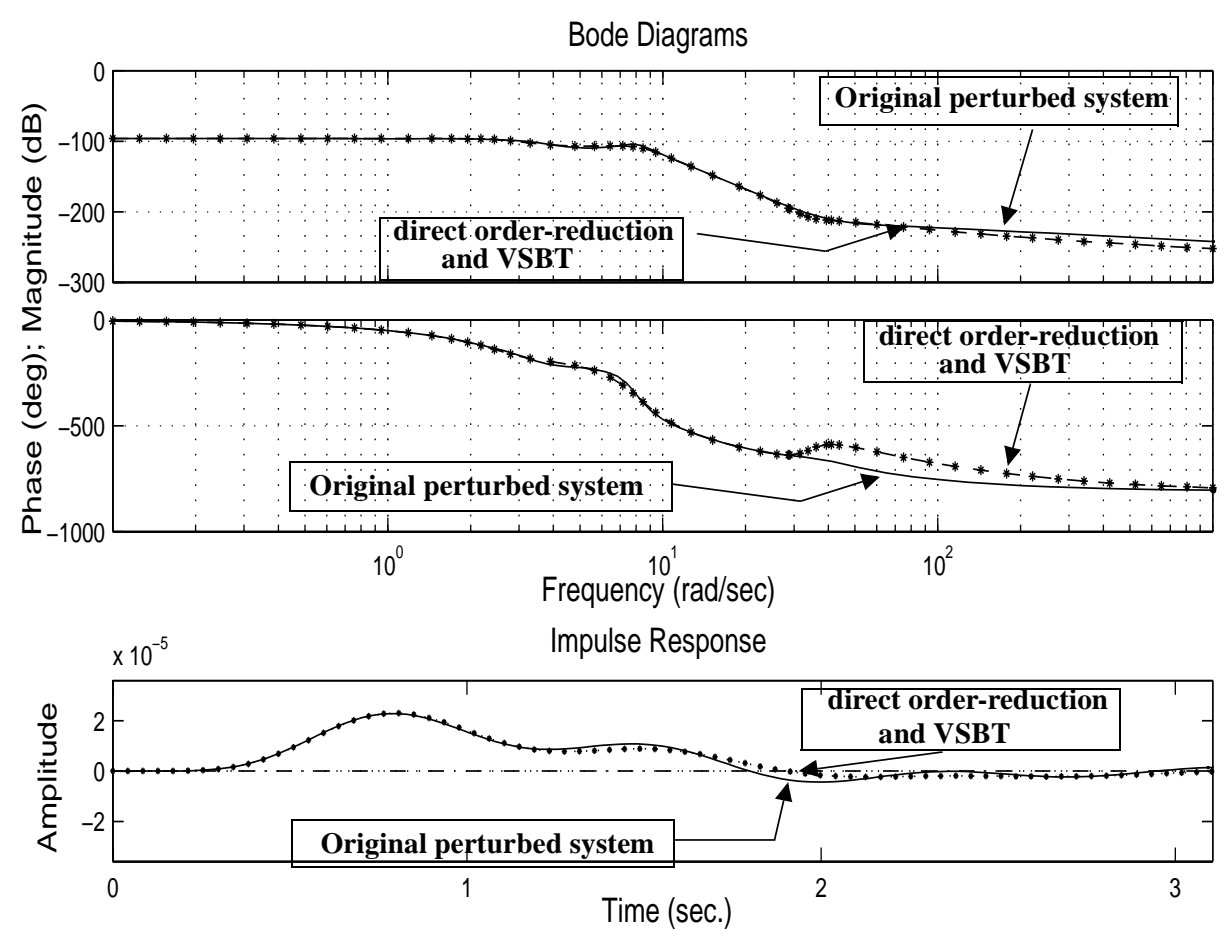

Fig. 11. The bode diagram and impulse response of the original perturbed system, the system obtained by direct spectrally-weighted balanced truncation, and the VSBT algorithm

\section{Conclusions and Future Work}

In this paper a variational spectrally-weighted balanced truncation technique for the model-order reduction of geometrically varying multiport RLC interconnects was proposed. It was shown that the balanced truncation technique is a highly effective approach when the variations in the circuit parameters need to be taken into consideration. Various experiments demonstrate that the computational run-time of the variational spectrally-weighted balanced truncation approach is $10-15 \%$ higher than that of the variational Krylov-subspace-based model-order reduction techniques while the accuracy is also $20 \%$ higher, on average.

As a future work, we will investigate the systematic way of determining the input/output weighting functions that minimize Eq. (13) in the spectrally-weighted balanced truncation method.

\section{Acknowledgement}

The authors would thank Prof. V. Sreeram at the Department of Electrical and Electronic Engineering, University of Western Australia, for the helpful discussions. 


\section{References}

[1] S. Oh, W. Jung, J. Kong, K. Lee, "Interconnect Modeling for VLSI," International Conf. on Simulation of Semiconductor Processes and Devices, pp. 203-206, 1999.

[2] L. T. Pillage, R. A. Rohrer, "Asymptotic Waveform Evaluation for Timing Analysis", IEEE Trans. CAD, vol. 9, No. 4, pp. 352-366, April 1990.

[3] C. Ratzlaff, L. T. Pillage, "RICE: Rapid Interconnect Circuit Evaluation Using AWE," IEEE Trans. CAD, vol. 13, No. 6, pp. 763-776, June 1994.

[4] K. J. Kerns, A. T. Yang, "Stable and Efficient Reduction of Large, Multiport RC Networks by Pole Analysis via Congruence Transformations," IEEE Trans. CAD, vol. 16, pp. 734-744, 1997.

[5] P. Feldmann, R. W. Freund, "Efficient Linear Circuit Analysis by Pade Approximation via the Lanczos Process,” IEEE Trans. CAD, vol. 14, pp. 639-649, May 1995.

[6] A. Odabasioglu, M. Celik, L. T. Pileggi, "PRIMA: Passive Reduced-Order Interconnect Macromodeling Algorithm," IEEE Trans. CAD, vol. 17, No. 8, pp. 645-654, Aug. 1998.

[7] J. Roychowdhury, "Reduced-Order Modeling of Time-Varying Systems," IEEE Trans. on Circuits and Systems II: Analog and Digital Signal Processing, pp. 1273-1288, vol. 46 , no. 10 , Oct. 1999.

[8] Li Peng, L. T. Pileggi, “NORM: Compact Model Order Reduction of Weakly Nonlinear Systems," IEEE/ACM Design Automation Conference, 2003. pp. 427-477, June 2003.

[9] K. Glover, All Optimal Hankel-norm Approximation of Linear Multivariable Systems and Their $L^{\infty}$-error Bounds," Int. J. Control, vol. 39, No. 6, pp. 1115-1193, 1984.

[10] P. Rabiei, M. Pedram, "Model Order Reduction of Large Circuits Using Balanced Truncation,” IEEE Proc. ASP-DAC, pp. 237-240, 1999.

[11] J. Li, F. Wang, J. White, “An Efficient Lyapunov Equation-Based Approach Generating Reduced-Order Models of Interconnect," 36th ACM/IEEE Design Automation Conference, pp. 16, 1999.

[12] J. Li, J. White, "Efficient Model Reduction of Interconnect via Approximate System Grammians," IEEE/ACM Proc. ICCAD, pp. 380-384, 1999.

[13] J. R. Phillips, L. Daniel, L. M. Silveira, "Guaranteed Passive Balancing Transformations for Model Order Reduction,” IEEE Trans. on CAD, pp. 1027- 1041, vol. 22, no. 8, Aug. 2003.

[14] A. Lu, E. L. Wachspress, "Solution of Lyapunov Equations by Alternating Direction Implicit," Computers and Mathematics with Applications, vol. 21, No. 9, pp. 43-58, June 1991. 
[15] N. Ellner, E. L. Wachspress, "Alternating Direction Implicit Iteration for Systems with Complex Spectra”, SIAM J. Numer., Anal., vol. 28, no. 3, pp. 859-870, June 1991.

[16] G. Wang, V. Sreeram, W. Q. Liu, “A New Frequency-Weighted Balanced Truncation Method and An Error Bound," IEEE Trans. on Automatic Control, pp. 1734 - 1737, vol. 44 , no. 9, Sept. 1999.

[17] V. Sreeram, "On the Properties of Frequency Weighted Balanced Truncation Techniques," Proc. American Control Conference, pp. 1753-1754, vol. 3 , May 2002.

[18] P. Heydari, M. Pedram "Balanced Truncation with Spectral Shaping for RLC Interconnects," IEEE Proc. ASP-DAC, pp. 203-208, Jan. 2001.

[19] Y. Liu, L. T. Pileggi, A. Strojwas, "Model Order-Reduction of RC(L) Interconnect including Variational Analysis," 36th IEEE/ACM Proc. Design Automation Conf., pp. 201-206, June 1999.

[20] G. H. Golub, C. Van Loan, Matrix Computation, pp. 390-405, Johns Hopkins University Press, 1996.

[21] P. Heydari, M. Pedram, "Model Reduction of Variable Geometry Interconnects Using Variational Spectrally-Weighted Balanced Truncation," IEEE Proc. ICCAD, San Jose, CA, Nov. 2001.

[22] L. Daniel, C. S. Ong, S. C. Low, K. H. Lee, J. White, A Multiparameter Moment Matching Model Reduction Approach for Generating Geometrically Parameterized Interconnect Performance Models", IEEE Trans. on Computer-Aided Design of Integrated Circuits and Systems, vol. 23, no. 5, pp. 678-93, May 2004.

[23] M. G. Safanov, R. Y. Chiang, "A Schur Method for Balanced Truncation Model Reduction," American Control Conference, vol. 2, pp. 1036-1040, 1988.

[24] P. J. Antsaklis, A. N. Micel, Linear Systems, New York: McGraw- Hill, 1997.

[25] O. S. Nakagawa, S. -Y. Oh, G. Ray, Modeling of Pattern-Dependent On-Chip Interconnect Geometry Variation of Deep-Submicron Process and Design Technology," IEEE International Technical Digest On Electron Devices Meeting, pp. 137-141, 1997.

[26] O. S. Nakagawa, N. Chang, S. Lin, D. Sylvester, "Circuit Impact and Skew Corner Analysis of Stochastic Process Variation in Global Interconnect," IITC, pp. 230-232, 1999.

[27] S. R. Nassif,"Modeling and Forecasting of Manufacturing Variations," 5th Int'l Workshop Statistical Metrology, pp. 2-10, 2000.

[28] Y. I. Ismail, E. G. Friedman, J. L. Neves, "Equivalent Elmore delay for RLC trees," IEEE/ ACM Design Automation Conference, pp. 715-720, June 1999. 